
\title{
Research S Surate \\ A Consensus Time for Performing Quality Control of 225Ac-Labeled Radiopharmaceuticals
}

James M. Kelly

Cornell University Joan and Sanford I Weill Medical College

Alejandro Amor-Coarasa

Yeshiva University Albert Einstein College of Medicine

Elizabeth Sweeney

Cornell University Joan and Sanford I Weill Medical College

John Babich ( $\square$ jwbabich@gmail.com )

Weill Cornell Medical College https://orcid.org/0000-0002-1616-3416

\section{Methodology}

Keywords: Target alpha therapy, Ac-225, radiopharmacy, quality control

Posted Date: July 2nd, 2020

DOI: https://doi.org/10.21203/rs.3.rs-39342/v1

License: (c) (1) This work is licensed under a Creative Commons Attribution 4.0 International License.

Read Full License 


\section{A Consensus Time for Performing Quality Control of ${ }^{225}$ Ac-Labeled Radiopharmaceuticals}

James M. Kelly, ${ }^{1}$ Alejandro Amor-Coarasa, ${ }^{2}$ Elizabeth Sweeney, ${ }^{3}$ John W. Babich ${ }^{1,4,5,}{ }^{*}$

${ }^{1}$ Molecular Imaging Innovations Institute (MI3) and Department of Radiology, Weill Cornell Medicine, New York, NY 10065, USA

${ }^{2}$ Department of Radiology, Albert Einstein College of Medicine, Bronx, NY 10461, USA

${ }^{3}$ Division of Biostatistics, Department of Population Health Sciences, Weill Cornell Medicine, New York, NY 10065, USA

${ }^{4}$ Sandra and Edward Meyer Cancer Center, Weill Cornell Medicine, New York, NY 10065, USA

${ }^{5}$ Citigroup Biomedical Imaging Center, Weill Cornell Medicine, New York, NY 10021, USA

*Corresponding Author:

John W. Babich, Ph.D.

Department of Radiology

Weill Cornell Medicine

Belfer Research Building

Room 1600

413 E $69^{\text {th }}$ St

New York, NY 10021

job2060@med.cornell.edu

Word Count (incl. References but excl. Supporting Information): 6854/6000 


\begin{abstract}
Background: As ${ }^{225} \mathrm{Ac}$-labeled radiopharmaceuticals continue to show promise as targeted alpha therapeutics, there is a growing need to standardize quality control (QC) testing procedures. The determination of radiochemical purity (RCP) is an essential $Q C$ test. A significant obstacle to RCP testing is the disruption of the secular equilibrium between actinium-225 and its daughter radionuclides during labeling and analysis. In order to accelerate translation of actinium-225 targeted alpha therapy, we aimed to determine the earliest time point at which the RCP of an ${ }^{225} \mathrm{Ac}$-labeled radiopharmaceutical can be accurately calculated.
\end{abstract}

Results: Six ligands were conjugated to macrocyclic metal chelators and labeled with actinium-225 under conditions designed to generate diverse incorporation yields. RCP was determined by radio thin layer chromatography (radioTLC) followed by exposure of the TLC plate on a phosphor screen either 0.5, 2, 3.5, $5,6.5$, or $26 \mathrm{~h}$ after the plate was developed. The dataset was used to create models for predicting the true RCP using pre-equilibrium measurements at early time points. The 585 TLC measurements span RCP values of $1.8 \%$ to $99.5 \%$. The statistical model created from these data predicted an independent data set with high accuracy. Predictions made at $0.5 \mathrm{~h}$ are more uncertain than predictions made at later time points. This is primarily due to the decay of bismuth-213. At $2 \mathrm{~h}$ the mean average error is $<3 \%$. A measurement of RCP $>90 \%$ at this time point predicts a true RCP $>97 \%$.

Conclusions: RCP of ${ }^{225} \mathrm{Ac}$-labeled radiopharmaceuticals can be quantified with acceptable accuracy at least $2 \mathrm{~h}$ after radioTLC. This time point best balances the need to accurately quantify RCP with the need to safely release the batch as quickly as possible.

Word Count: $279 / 350$

Keywords: Target alpha therapy, Ac-225, radiopharmacy, quality control 


\section{Introduction}

Targeted alpha-particle therapy (TAT) shows great promise in the treatment of cancer. Following the successful introduction of bone-avid ${ }^{223} \mathrm{RaCl}_{2}\left(\right.$ Xofigo $\left.^{\circledR}\right)$ for the treatment of skeletal metastases (1-3), TAT using astatine-211 (4,5), lead-212 (6), bismuth-213 (7-9), and actinium-225 $(10,11)$ has been investigated for the treatment of multiple cancers. Actinium-225 decays by emission of four alpha particles with a halflife $\left(t_{1 / 2}=9.9 \mathrm{~d}\right)$. These physical properties $(12,13)$ and growing supply $(14)$ contribute to an increasing interest in the use of actinium-225 for TAT. Clinical evaluations of $\left[{ }^{225} A c\right] A c-D O T A T O C ~(15),\left[{ }^{225} A c\right] A c-$ PSMA-617 (16-18), and [ ${ }^{225}$ Ac]AC-DOTA-Substance $P(19)$ report significantly improved responses in patients with neuroendocrine tumors, prostate cancer, and glioma. Dramatic responses are even observed in patients refractory to beta-particle therapy (11). In addition to these small molecule radioligands, $\left[{ }^{225} \mathrm{Ac}\right] \mathrm{Ac}-\mathrm{J} 591$ is currently in Phase I clinical trials for radioimmunotherapy of prostate cancer (20). Given these early and promising findings the clinical development of actinium-225 TAT will likely continue to grow.

Actinium-225 is introduced to tumor-targeting vectors through its complexation by a chelating moiety. This is most commonly achieved using bifunctional derivatives of 1,4,7,10-tetraazacyclododecane$1,4,7,10$-tetraacetic acid (DOTA) $(15,16,19,21)$ conjugated to the vector, although new bifunctional macrocyclic chelators that complex actinium-225 with high specificity and stability have also been recently reported $(22,23)$. The preparation of ${ }^{225} \mathrm{Ac}$-labeled radiopharmaceuticals typically requires a period of

incubation of the precursor ligand and an actinium-225 salt (e.g., $\left[{ }^{225} \mathrm{Ac}\right] \mathrm{AcCl}_{3}$ or $\left.\left[{ }^{225} \mathrm{Ac}\right] \mathrm{Ac}\left(\mathrm{NO}_{3}\right)_{3}\right)$ at acidic or neutral $\mathrm{pH}$, followed by a solvent exchange and sterilization of the radiopharmaceutical solution. In some cases, purification of the radiolabeled compound from uncomplexed actinium-225 may be required.

Each batch production of a radiopharmaceutical for clinical use must undergo quality control (QC) testing prior to release. A critical release parameter is radiochemical purity (RCP); the proportion of the total radioactivity in a sample which is present as the desired radiolabeled species. Currently, the RCP of ${ }^{225} \mathrm{Ac}-$ labeled radiopharmaceuticals is primarily assessed using radio thin-layer chromatography (radioTLC) and a mobile phase that effectively separates non-complexed radiometal (and radiolabeled impurities) from the desired radiopharmaceutical. However, the complex decay chain of actinium-225 renders quantification of RCP challenging because daughter radionuclides may not be complexed and may distribute across the TLC plate in a manner that overlaps with the radiopharmaceutical. Highly accurate quantification of RCP for ${ }^{225} \mathrm{Ac}$-labeled radiopharmaceuticals requires secular equilibrium to be reached between actinium-225 and its daughter radionuclides. Secular equilibrium is reached after $20 \mathrm{~h}(24,25)$, but such a long analysis window is impractical in a clinical production setting. Consequently, sites producing ${ }^{225} \mathrm{Ac}$-labeled radiopharmaceuticals wait between $1 \mathrm{~h}$ and $12 \mathrm{~h}$ after running the TLC plate before quantifying $\operatorname{RCP}(11,23,26,27)$. This site-specific practice introduces variability into the radiopharmaceutical production process. Ultimately, this may lead to confounding results when clinical evaluations are compared across multiple sites and potentially delay approval of the radiopharmaceutical by the governing regulatory agency.

To work toward a consensus quality control protocol for ${ }^{225} \mathrm{Ac}$-labeled radiopharmaceuticals, we aimed to determine the earliest time at which analysis of the TLC plate can be performed with high predictive 
accuracy. Herein we present a statistical analysis of over 500 radioTLC results performed at various time points. Using this analysis, we predict the RCP at secular equilibrium for radiopharmaceuticals bearing either bifunctional DOTA or bifunctional N,N'-bis[(6-carboxy-2-pyridyl)methyl]-4,13-diaza-18-crown-6 (macropa) chelating moieties from measurements taken before equilibrium is reached. Our model proposes a suitable time at which the RCP of ${ }^{225} \mathrm{Ac}$-labeled radiopharmaceuticals can be predicted with sufficient accuracy as to permit batch release.

\section{Materials and Methods}

\section{Radiolabeling Experiments}

In order to generate a diverse range of radiochemical yields, reactions were performed by varying the precursor (chelator) and precursor concentration, and the reaction time and temperature. Reactions were performed in triplicate. Six small molecule ligands targeting prostate-specific membrane antigen (PSMA), RPS-072 (28), RPS-074 (29), EuK-106 (30), EuK-107 (30), RPS-088, and RPS-092 (Figure S1), were prepared as stock solutions of $0.01-1 \mathrm{mg} / \mathrm{mL}$ in DMSO. Actinium-225 (9.25 MBq) was obtained from Canadian National Laboratories as the dried $\left[{ }^{225} \mathrm{Ac}\right] \mathrm{AcCl}_{3}$ salt. The $\left[{ }^{225} \mathrm{Ac}\right] \mathrm{AcCl}_{3}$ was dissolved in $1 \mathrm{~mL} 1 \mathrm{M} \mathrm{NH}{ }_{4} \mathrm{OAc}$, $\mathrm{pH} 7.0$, transferred by pipette to a $50 \mathrm{~mL}$ centrifuge tube (Corning), and diluted to $45 \mathrm{~mL}$ in $1 \mathrm{M} \mathrm{NH}_{4} \mathrm{OAc}$. One $\mathrm{mL}$ of the stock solution, containing approximately $205 \mathrm{kBq}\left[{ }^{225} \mathrm{Ac}\right] \mathrm{Ac}(\mathrm{OAc})_{3}$, was transferred by pipette to a plastic Eppendorf tube placed on a digital ThermoMixer (Eppendorf) heating block. Then 20 $\mu \mathrm{L}$ of the ligand stock solution (0.01-1 $\mathrm{mg} / \mathrm{mL}$ in DMSO) was added and the reaction was shaken at 300 $\mathrm{rpm}$ at either $25^{\circ} \mathrm{C}$ or $95^{\circ} \mathrm{C}$ for $15 \mathrm{~min}$ (Table 1). A $3 \mu \mathrm{L}$ aliquot of the reaction mixture was withdrawn and deposited on the origin of a silica gel 60-coated aluminum plate (Sigma Aldrich) after incubating the reaction for $1 \mathrm{~min}, 5 \mathrm{~min}$, and $15 \mathrm{~min}$.

\begin{tabular}{|c|c|c|c|}
\hline Compound & Chelator & [Compound] $(\mu \mathrm{M})$ & $\mathrm{T}\left({ }^{\circ} \mathrm{C}\right)$ \\
\hline RPS-072 & Macropa, DOTA & 9.0 & 95 \\
\hline \multirow{3}{*}{ RPS-074 } & \multirow{3}{*}{ Macropa } & 8.9 & \multirow{3}{*}{25} \\
\hline & & 0.89 & \\
\hline & & 0.089 & \\
\hline \multirow{3}{*}{ RPS-088 } & \multirow{3}{*}{ Macropa } & 10.4 & \multirow{3}{*}{25} \\
\hline & & 1.04 & \\
\hline & & 0.104 & \\
\hline \multirow{2}{*}{ RPS-092 } & \multirow{2}{*}{ Macropa } & 8.4 & \multirow{2}{*}{25} \\
\hline & & 0.84 & \\
\hline EuK-106 & DOTA-106 & 18.2 & 95 \\
\hline EuK-107 & DOTA-107 & 20.0 & 95 \\
\hline
\end{tabular}

Table 1. Reaction conditions for radiolabeling. Conditions were selected to increase diversity rather than to maximize radiochemical yield. The chelator associated with each molecule is indicated. Full structures are provided in Figure S1.

\section{Analysis of Reaction Yield}

After the final aliquot was deposited, the plate was immediately run in freshly prepared mobile phase comprised of $10 \% \mathrm{v} / \mathrm{v} \mathrm{MeOH} / 10 \mathrm{mM}$ EDTA (in $\mathrm{H}_{2} \mathrm{O}$ ). A separate plate containing a $3 \mu \mathrm{L}$ aliquot of the 
solution of $\left[{ }^{225} \mathrm{Ac}\right] \mathrm{Ac}(\mathrm{OAc})_{3}$ in $1 \mathrm{M} \mathrm{NH}_{4} \mathrm{OAc}$ was run as a control. The actinium-225 was administered as the triacetate salt, but transchelation by EDTA to form [ $\left.{ }^{225} \mathrm{Ac}\right] \mathrm{Ac}$-EDTA is likely. The plates were developed until the solvent front had traveled at least $60 \%$ of the total plate length (35-45 min). Upon removal from the mobile phase, the plates were dried and visualized at $0.5 \mathrm{~h}, 2 \mathrm{~h}, 3.5 \mathrm{~h}, 5 \mathrm{~h}, 6.5 \mathrm{~h}$, and $26 \mathrm{~h}$ intervals using a Cyclone Plus Storage Phosphor System (Perkin Elmer) following a 2 min direct exposure on a phosphor screen. The screen was erased with homogenous white light for $5 \mathrm{~min}$ between exposures. The retention factors, $R_{f}$, of the radiolabeled compounds were 0.0-0.3, while [ $\left.{ }^{225} A c\right]$ Ac-EDTA (and daughter radionuclides present at time of spotting) migrated mainly toward the solvent front $\left(R_{f}>0.7\right)$. Labeling yield was determined using OptiQuant ${ }^{\mathrm{TM}}$ software (Perkin Elmer) by fitting a boxed grid to the plate and quantifying the counts in all regions. Radiochemical yield was defined as the ratio of the counts in the box corresponding to the labeled product to the sum of the counts in all boxes (Figure 1). Experimentallydetermined radiolabeling yield was plotted as a function of analysis time using GraphPad Prism 8 (GraphPad Software).

Statistical predictive modeling:

All statistical modeling was performed in the R environment (version 3.5.0, R Foundation for Statistical Computing, Vienna, Austria). Ninety-nine observation sets ( 11 initial reaction conditions $x 3$ reaction times $x 3$ replicates) were randomly assigned to training and validation sets (66 sets to the training set and 33 sets to the validation set). As the labeling percentage for the samples were bounded between $0(0 \%)$ and $1(100 \%)$, a generalized linear model with binomial link was fit to the data in the training set to model the relationship between the empirically-measured yield at $26 \mathrm{~h}$ (defined as the true RCP) and the time of each observation, the yield of the observation at that time point, and the interaction between the two. Samples with a value of 0.9 or more at $26 \mathrm{~h}$ were weighted 10 times more than the other samples in the model in order to optimize our model for predictions of values in this area.

Predictions from the model were made in the validation set and mean absolute error (MAE) was calculated in the validation set for strata of each time (predictions using data at $0.5 \mathrm{~h}, 2 \mathrm{~h}, 3.5 \mathrm{~h}, 5 \mathrm{~h}$, and $6.5 \mathrm{~h}$ ). We also report a predicted outcomes table, where the generalized linear model was retrained using the entire dataset, continuing to weight the samples in the manner described above. The predicted RCP at $26 \mathrm{~h}$ from the model for each of the measured values is reported as well as $95 \%$ prediction intervals.

We fit a random forest classification model $(31,32)$ on the training set to predict labeling percentage above a given threshold. The predictors in this model include the time that a given observation is made and the yield of that observation. Models were fit using a discrimination threshold of 0.9 (90\%) or 0.95 (95\%). For a threshold of 0.9 , there were 140 observations $\geq 0.9$ in the data set, and 190 observations $<0.9$. For a threshold of 0.95 , there were 125 observations $\geq 0.95$ in the data set, and 205 observations $<0.95$. A receiver operating characteristic (ROC) curve is plotted for each classification model in the validation set, and area under the curve (AUC) and $95 \%$ confidence interval $(\mathrm{Cl})$ are reported.

The model was validated using an independent data set consisting of observations made at $0.5,1.5,2.0$, 2.5 , and $26 \mathrm{~h}$. The MAE of the predictions are reported at each time point.

\section{Mathematical modeling}


The decay of actinium-225 and its daughter nuclei was modeled based on ten general assumptions:

1. All low probability emissions in the actinium-225 decay chain (Figure $1 \mathrm{~A}$ ) were disregarded (Figure 1B).

2. The detection efficiency of $\beta$ and $\alpha$ emissions by the phosphor screen was considered to be equal.

3. $\left[{ }^{225} \mathrm{Ac}\right] \mathrm{Ac}^{3+}$ and $\left[{ }^{213} \mathrm{Bi}^{3} \mathrm{Bi}^{3+}\right.$ are complexed equivalently by DOTA. This assumption is based upon the suggestion that the two complexes have similar association (33) and dissociation kinetics (34) and the fact that DOTA is present in significant excess with respect to the metal ions.

4. Macropa does not chelate $\left[{ }^{213} \mathrm{Bi}^{-} \mathrm{Bi}^{3+}\right.$ as efficiently as it does $\left[{ }^{225} \mathrm{Ac}\right] \mathrm{Ac}^{3+}$. This assumption is based upon the size selectivity of macropa towards metals with larger atomic radii (35).

5. There are at least 320 times more actinium-225 atoms than bismuth-213 atoms in solution at any given time point, therefore $99 \%$ of the bismuth- 213 is bound by DOTA in the time that $1 \%$ of the actinium-225 is chelated.

6. At time 0 after spotting and before running the TLC, all the activity is at the origin.

7. The TLC plate can be divided into three sections, representing the radiolabeled complex (1, Figure $1 C$ ), $\left[{ }^{225} \mathrm{Ac}\right] \mathrm{Ac}(\mathrm{OAc})_{3}$ (or $\left[{ }^{225} \mathrm{Ac}\right] \mathrm{Ac}-\mathrm{EDTA}$ ) that was not bound at the time of spotting (3, Figure $1 \mathrm{C}$ ), and a mixture of $\left[{ }^{225} \mathrm{Ac}\right] \mathrm{Ac}-\mathrm{EDTA}$ that did not run homogenously and daughter nuclei emitted while the plate was running (2, Figure $1 \mathrm{C})$. The content of these sections was determined experimentally by radioTLC analysis of the $\left[{ }^{225} \mathrm{Ac}\right] \mathrm{Ac}(\mathrm{OAc})_{3}$ control.

8. All metal ions that were uncomplexed at the time of spotting migrated with the solvent front $\left(R_{f}\right.$ $>0.7)$.

9. The two main radioactive equilibriums governing the activity distribution on the TLC plate are those established by actinium-225 and bismuth-213 when purified from their precursors.

10. Lead-209 $\left(t_{1 / 2}=3.25 \mathrm{~h}\right)$ has a sufficiently long half-life that its contribution to radiochemical equilibrium must be taken into account. Consequently, each actinium-225 and bismuth-213 disintegration, which produces 6 and 3 particles, respectively, must be discounted due to the slower decay of lead-209 to bismuth-209.

For bismuth-213 in equilibrium: Counts/disintegration $=2.25 \times \mathrm{A}\left({ }^{213} \mathrm{Bi}\right)$

For actinium-225 in equilibrium: Counts/disintegration $=5.25 \times \mathrm{A}\left({ }^{225} \mathrm{Ac}\right)$
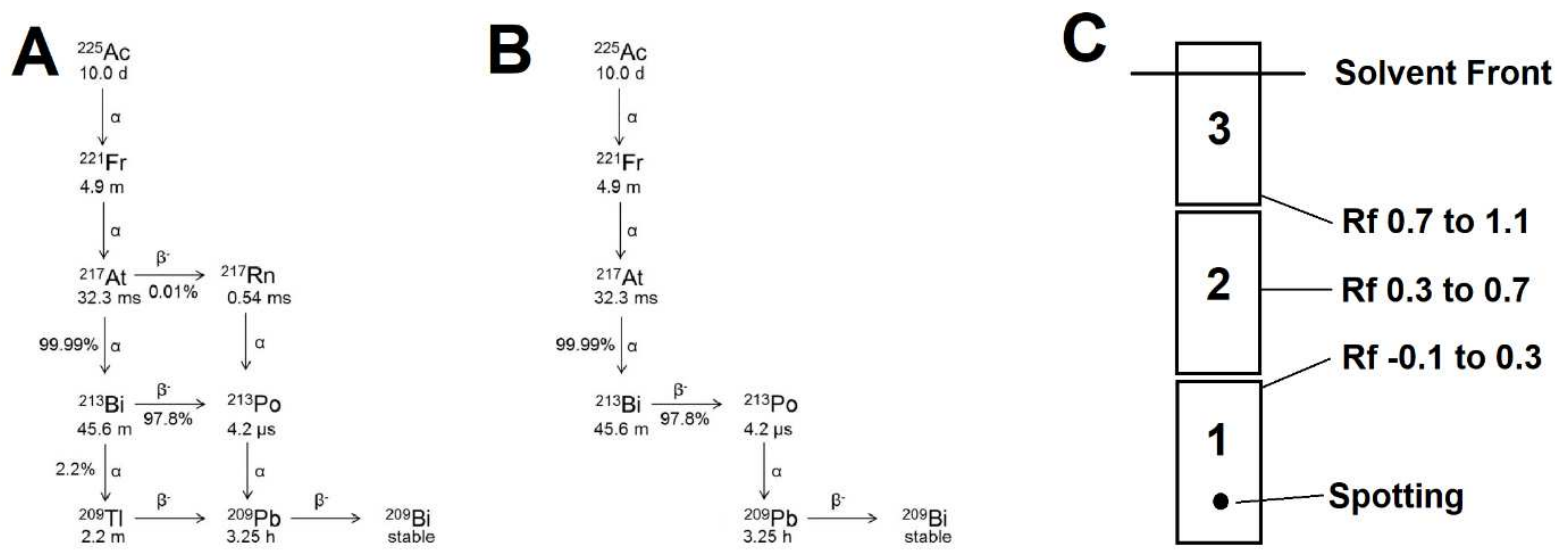
Figure 1. Approximations used for the mathematical model. A. Actinium-225 decay scheme. B. The actinium-225 decay scheme used for the purpose of the model. C. General representation of the box grid used to quantify radiolabeling by radioTLC.

Equations describing the expected activity in each grid of the TLC plate were derived and plotted using Origin (OriginLab). Expected activity distribution was determined by solving the equations at $0.5 \mathrm{~h}, 2 \mathrm{~h}$, $3.5 \mathrm{~h}, 5 \mathrm{~h}, 6.5 \mathrm{~h}$, and $26 \mathrm{~h}$. A 3-dimensional (3D) surface plot of analysis time versus experimentallydetermined radiolabeling yield was derived for each group of compounds sharing the same chelator moiety. Similar three-dimensional (3D) plots were prepared for analysis time versus mathematically modeled radiolabeling yield. Differences between experimentally-derived radiolabeling yield and modelpredicted yield were compared statistically by a paired t-test. A threshold of $p<0.05$ was used to define statistical significance.

\section{Results}

\section{Radiolabeling}

Mean radiolabeling yield after $1 \mathrm{~min}$, as assessed $26 \mathrm{~h}$ after running the TLC, ranged from $2.7 \pm 0.55 \%$ to $98.8 \pm 0.09 \%$. The highest radiochemical yields were obtained using macropa-conjugated RPS-074 and RPS-088, which exceeded $97 \%$ at a ligand concentration of $9-10 \mu \mathrm{M}$. The range after 5 min was $3.8 \pm 1.95 \%$ to $99.0 \pm 0.04 \%$, and the range after 15 min was $11.2 \pm 5.25 \%$ to $99.2 \pm 0.32 \%$. RPS-074 and RPS-088 were rapidly labeled at $25^{\circ} \mathrm{C}$, and there was no significant change in labeling yield when ligand concentration decreased from approximately $10 \mu \mathrm{M}$ to approximately $1 \mu \mathrm{M}$. At a ligand concentration of approximately $0.1 \mu \mathrm{M}$, radiolabeling yield was highly variable, ranging from $5.9 \%$ to $92.1 \%$. EuK-107, bearing an amine analogue of the $3 \mathrm{p}-\mathrm{C}$-DEPA chelator, was rapidly labeled at $95^{\circ} \mathrm{C}$, while DOTA-containing RPS-072 reached $93.2 \pm 1.21 \%$ after $15 \mathrm{~min}$ at $95{ }^{\circ} \mathrm{C}$ and a ligand concentration of $9 \mu \mathrm{M}$. In agreement with previously published results (30), EuK-106 was poorly labeled (24.8 $\pm 2.36 \%$ ) even after $15 \mathrm{~min}$ at $95^{\circ} \mathrm{C}$.
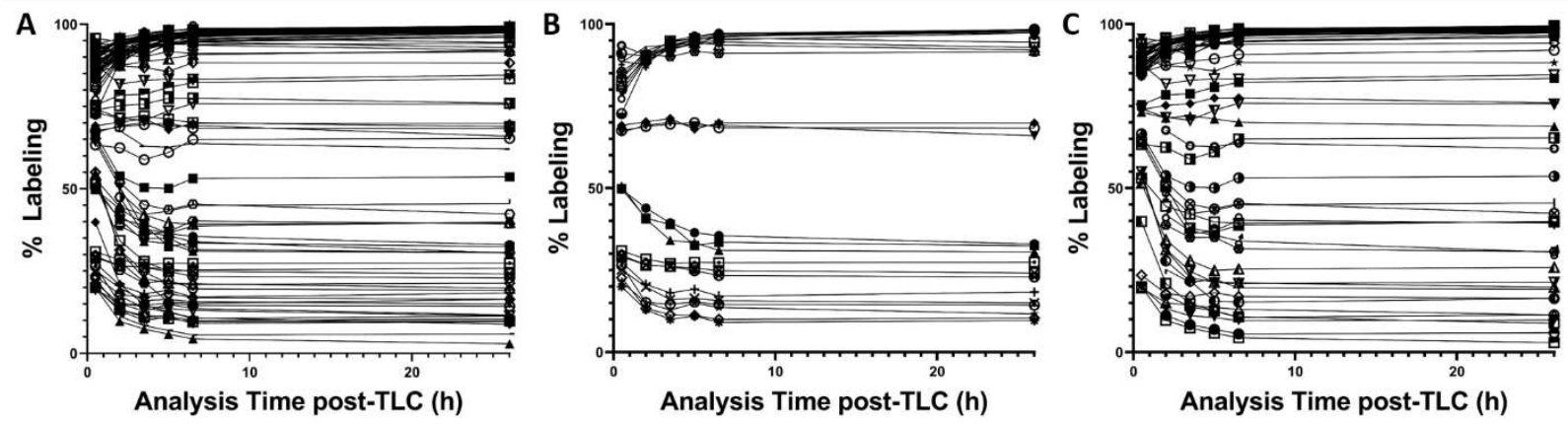

Figure 2. Plot of experimentally-derived radiolabeling versus analysis time after TLC for multiple reaction conditions. Radiolabeling was performed using RPS-072, RPS-074, RPS-088, RPS-092, EuK-106, or EuK-107 and $\left[{ }^{225} \mathrm{Ac}\right] \mathrm{AcCl}_{3}$ in $1 \mathrm{M} \mathrm{NH}_{4} \mathrm{OAc}$. Conditions are described in Table 1 . Activity distribution on the TLC plates was visualized at the specified times using a Cyclone Plus Storage Phosphor System. Quantification of labeling was performed using OptiQuant ${ }^{\mathrm{TM}}$ software. A. All Compounds. B. DOTA-containing ligands (RPS072, EuK-106, EuK-107). C. Macropa-containing ligands (RPS-074, RPS-088, RPS-092). 
A

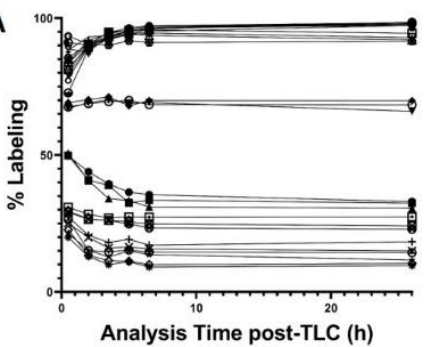

B

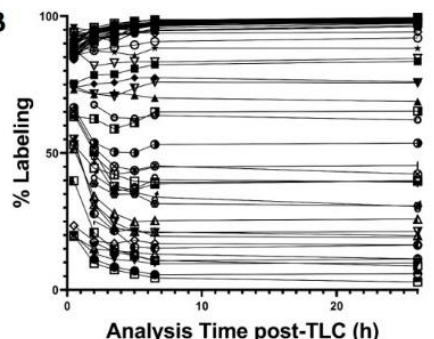

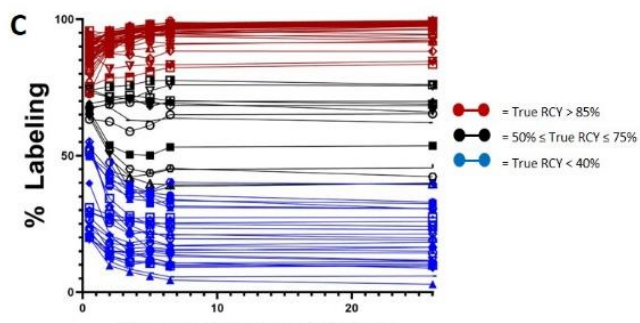

Analysis Time post-TLC (h)

[AA1]

Figure 2. Plot of experimentally-derived radiolabeling versus analysis time after TLC for multiple reaction conditions. Radiolabeling was performed using RPS-072, RPS-074, RPS-088, RPS-092, EuK-106, or EuK-107 and $\left[{ }^{225} \mathrm{Ac}_{\mathrm{AcCl}}\right.$ in $1 \mathrm{M} \mathrm{NH}_{4} \mathrm{OAc}$. Conditions are described in Table 1 . Activity distribution on the TLC plates was visualized at the specified times using a Cyclone Plus Storage Phosphor System. Quantification of labeling was performed using OptiQuant ${ }^{\mathrm{TM}}$ software. A. DOTA-containing ligands (RPS-072, EuK-106, EuK107). B. Macropa-containing ligands (RPS-074, RPS-088, RPS-092). C. All compounds.

Three clusters of radiolabeled compounds were observed (Figure 2). For those compounds that were efficiently labeled ( $>85 \%$ as determined at $26 \mathrm{~h}$ ), early analysis time underestimated radiolabeling yield (RCY). The radiolabeling of compounds labeled with moderate efficiency, $50 \% \leq \mathrm{RCY} \leq 75 \%$ at $26 \mathrm{~h}$, was estimated with reasonable accuracy at early analysis times. Finally, the labeling of compounds with low $\mathrm{RCY}(<40 \%$ at $26 \mathrm{~h})$, is overestimated at early analysis times.

\section{Statistical predictive modeling}

We developed a statistical model to predict the true RCP of an unpurified reaction based on pre-secularequilibrium measurements. The predicted RCP and true RCP are well matched for reactions with high yield $(R C Y \geq 85 \%)$ and low yield ( $R C Y \leq 25 \%)$. In the intermediate range $25 \% \leq R C Y \leq 85 \%$, predictions generally overestimate the true RCP (Figure 3). The accuracy of the predictions is lowest at $0.5 \mathrm{~h}$, at which time MAE is 0.053 (5.3\%). For TLC reads performed from $2 \mathrm{~h}$ onwards, the accuracy of the model is high. MAE is $3.2 \%$ at $2 \mathrm{~h}$, decreases to $2.6 \%$ at $3.5 \mathrm{~h}$ and $5 \mathrm{~h}$, and $2.9 \%$ at $6 \mathrm{~h}$. This indicates that labeling yield may be overor underestimated by no more than $3 \%$ after $2 \mathrm{~h}$. The analysis was also performed separately for the macropa-containing ligands RPS-074, RPS-088, and RPS-092, and for the DOTA-containing ligands RPS072, EuK-106, and EuK-107. Neither sub-analysis differed significantly from the analysis using the entire data set (Figure S2-S4, Tables S1, S2). 


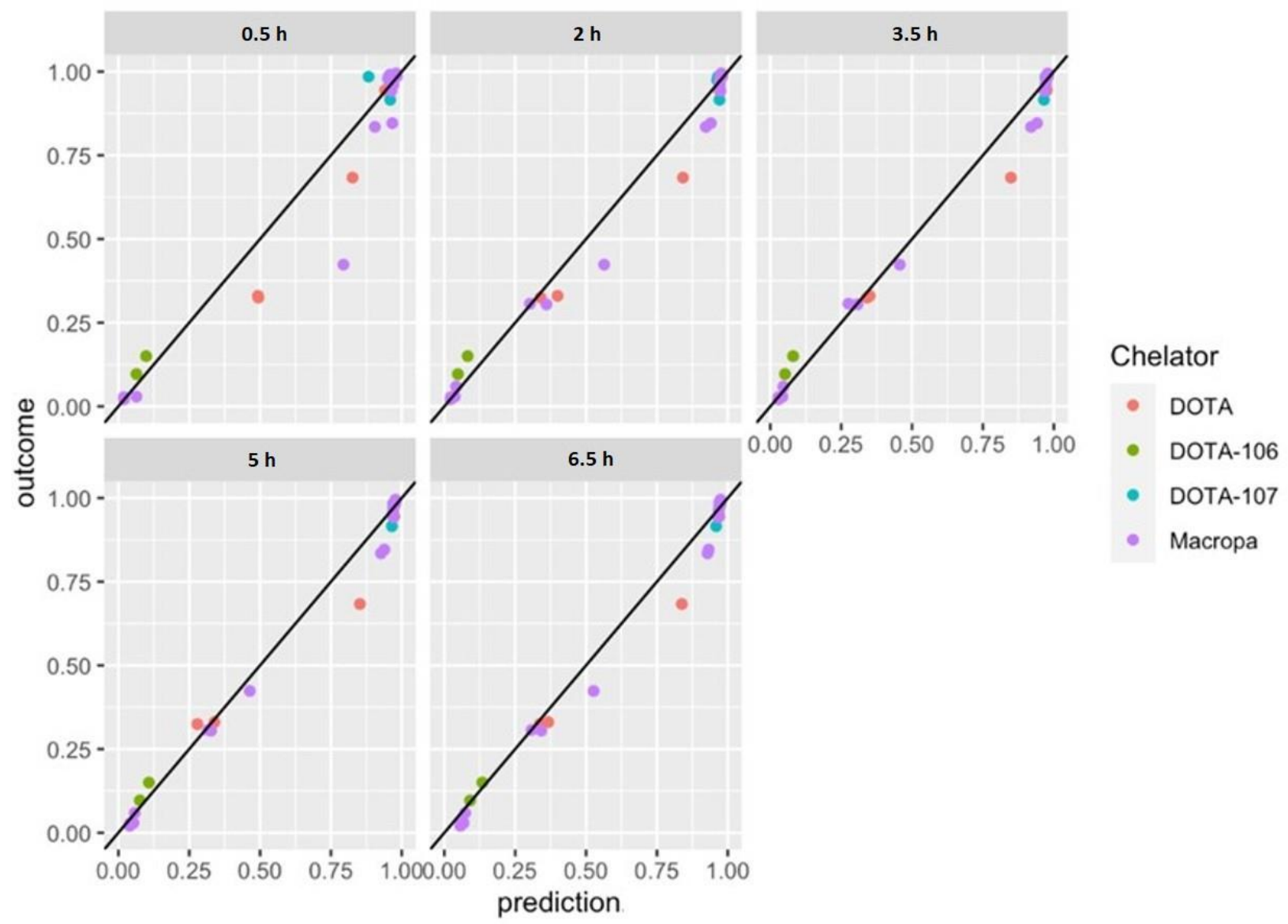

Figure 3. Predictive accuracy of $Q C$ measurements taken at $0.5 \mathrm{~h}, 2 \mathrm{~h}, 3.5 \mathrm{~h}, 5 \mathrm{~h}$, or $6.5 \mathrm{~h}$ after running the radioTLC plate. X-axis: Predicted radiochemical yield. Y-axis: True radiochemical yield, defined as the radiochemical yield when measured at $26 \mathrm{~h}$ after running the TLC.

The model was validated using an independent data set collected for radiolabeling of a macropaconjugated antibody (Figure S5). The predictions of the model were highly accurate, with an MAE of $1.3 \%$ at $0.5 \mathrm{~h}$ that decreases to $0.9 \%, 0.8 \%$, and $0.8 \%$ at $1 \mathrm{~h}, 1.5 \mathrm{~h}$, and $2 \mathrm{~h}$, respectively (Table S3). We therefore used our statistical model to predict the true RCP on the basis of TLC measurements at earlier time points. Our model predicts that labeling yield in excess of $90 \%$, as measured at any time point, is highly likely to translate to a true labeling yield greater than 95\% (Table 2). However, range of expected values at the 30 min time point for $\mathrm{RCY} \leq 97 \%$ includes a lower bound $<95 \%$. This means that a measurement of $97 \%$ at 30 min could possibly result in a true value $<95 \%$. By $2 \mathrm{~h}$, the range of expected values is $95-100 \%$ for all $\mathrm{RCY} \geq 94 \%$. This implies that a purity threshold of $95 \%$ for batch release will be definitively met if the yield is measured to be $\geq 98 \%$ at $0.5 \mathrm{~h}$ or $\geq 94 \%$ at $2 \mathrm{~h}$ after running the TLC plate. The expanded prediction intervals at the endpoints of the model reflect the absence of data for $t<0.5 \mathrm{~h}$ and $t>6.5 \mathrm{~h}$, which results in inaccurate extrapolation. 


\begin{tabular}{|c|c|c|c|c|c|c|c|c|c|}
\hline $\begin{array}{c}\text { RCY AT } \\
0.5 \mathrm{~h} \\
(\%)\end{array}$ & $\begin{array}{c}\text { EXPECTED } \\
\text { RCY (\%) }\end{array}$ & $\begin{array}{c}\text { RCY } \\
\text { at } 2 \mathrm{~h} \\
(\%)\end{array}$ & $\begin{array}{c}\text { EXPECTED } \\
\text { RCY (\%) }\end{array}$ & $\begin{array}{c}\text { RCY at } \\
3.5 \mathrm{~h} \\
(\%)\end{array}$ & $\begin{array}{c}\text { EXPECTED } \\
\text { RCY (\%) }\end{array}$ & $\begin{array}{c}\text { RCY at } \\
5 \text { h } \\
(\%)\end{array}$ & $\begin{array}{c}\text { EXPECTED } \\
\text { RCY (\%) }\end{array}$ & $\begin{array}{c}\text { RCY at } \\
6.5 \mathrm{~h} \\
(\%)\end{array}$ & $\begin{array}{c}\text { EXPECTED } \\
\text { RCY (\%) }\end{array}$ \\
\hline 0 & $1(0,23)$ & 0 & $2(0,14)$ & 0 & $2(0,12)$ & 0 & $4(1,17)$ & 0 & $5(0,39)$ \\
\hline 10 & $3(0,33)$ & 10 & $4(1,23)$ & 10 & $5(1,19)$ & 10 & $7(2,26)$ & 10 & $10(1,48)$ \\
\hline 20 & $6(1,45)$ & 20 & $8(2,34)$ & 20 & $11(3,30)$ & 20 & $14(4,37)$ & 20 & $17(3,58)$ \\
\hline 30 & $14(2,58)$ & 30 & $17(5,48)$ & 30 & $21(8,43)$ & 30 & $24(9,51)$ & 30 & $29(7,68)$ \\
\hline 40 & $29(7,70)$ & 40 & $32(12,62)$ & 40 & $36(18,58)$ & 40 & $40(20,64)$ & 40 & $44(16,76)$ \\
\hline 50 & $50(19,80)$ & 50 & $52(29,75)$ & 50 & $55(36,72)$ & 50 & $58(37,76)$ & 50 & $60(31,84)$ \\
\hline 60 & $71(43,89)$ & 60 & $72(53,85)$ & 60 & $73(58,84)$ & 60 & $74(57,86)$ & 60 & $75(50,90)$ \\
\hline 70 & $85(68,94)$ & 70 & $85(74,92)$ & 70 & $85(76,91)$ & 70 & $85(74,92)$ & 70 & $85(68,94)$ \\
\hline 80 & $93(84,98)$ & 80 & $93(87,97)$ & 80 & $93(87,96)$ & 80 & $92(85,96)$ & 80 & $92(80,97)$ \\
\hline 90 & $97(92,99)$ & 90 & $97(93,99)$ & 90 & $97(93,98)$ & 90 & $96(92,98)$ & 90 & $96(88,99)$ \\
\hline 91 & $97(92,99)$ & 91 & $97(93,99)$ & 91 & $97(94,98)$ & 91 & $96(92,98)$ & 91 & $96(88,99)$ \\
\hline 92 & $98(92,99)$ & 92 & $97(94,99)$ & 92 & $97(94,98)$ & 92 & $97(93,98)$ & 92 & $96(89,99)$ \\
\hline 93 & $98(93,99)$ & 93 & $98(94,99)$ & 93 & $97(94,99)$ & 93 & $97(93,99)$ & 93 & $96(89,99)$ \\
\hline 94 & $98(93,99)$ & 94 & $98(95,99)$ & 94 & $97(95,99)$ & 94 & $97(93,99)$ & 94 & $97(90,99)$ \\
\hline 95 & $98(94,100)$ & 95 & $98(95,99)$ & 95 & $98(95,99)$ & 95 & $97(94,99)$ & 95 & $97(90,99)$ \\
\hline 96 & $98(94,100)$ & 96 & $98(95,99)$ & 96 & $98(95,99)$ & 96 & $97(94,99)$ & 96 & $97(90,99)$ \\
\hline 97 & $98(94,100)$ & 97 & $98(95,99)$ & 97 & $98(96,99)$ & 97 & $98(94,99)$ & 97 & $97(91,99)$ \\
\hline 98 & $99(95,100)$ & 98 & $98(96,99)$ & 98 & $98(96,99)$ & 98 & $98(95,99)$ & 98 & $97(91,99)$ \\
\hline 99 & $99(95,100)$ & 99 & $99(96,99)$ & 99 & $98(96,99)$ & 99 & $98(95,99)$ & 99 & $98(92,99)$ \\
\hline 100 & $99(95,100)$ & 100 & $99(96,100)$ & 100 & $98(96,99)$ & 100 & $98(95,99)$ & 100 & $98(92,99)$ \\
\hline
\end{tabular}

Table 2. Predicted true labeling, defined as the labeling yield measured at $26 \mathrm{~h}$ after running the TLC, based on earlier measurements. Expressed as expected value with prediction interval.

We derived two classification models to further evaluate the accuracy of our predictions. With a discrimination threshold of 0.9 , which corresponds to $90 \%$ labeling yield, the predictive accuracy of the model is $99 \%$ (160/162) (Figure 4A). Both of the incorrect predictions are made at the $0.5 \mathrm{~h}$ analysis time point. An increase of the discrimination threshold to 0.95 results in a slight decrease in predictive accuracy to $91 \%$ (147/162) (Figure 4B). Much of the discrepancy between prediction and true outcome is due to "false positives" (14/162), in which the prediction overestimates the true yield. The AUC of both curves exceeds 0.95 , confirming the high accuracy of the tests.

A.

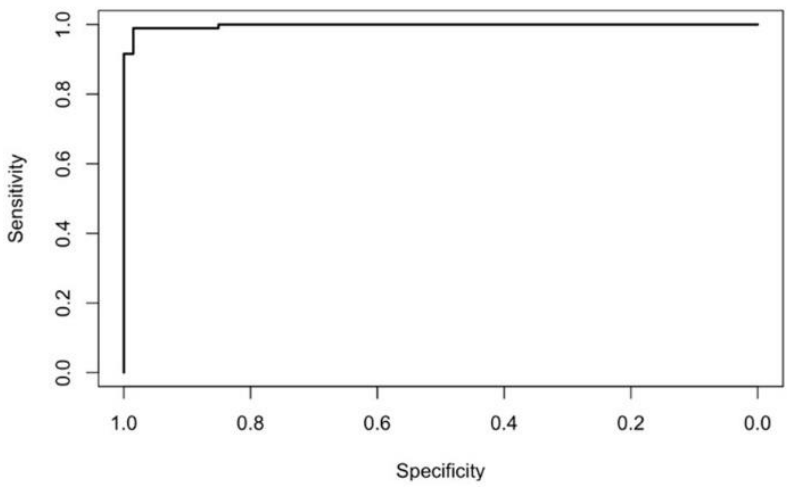

B.

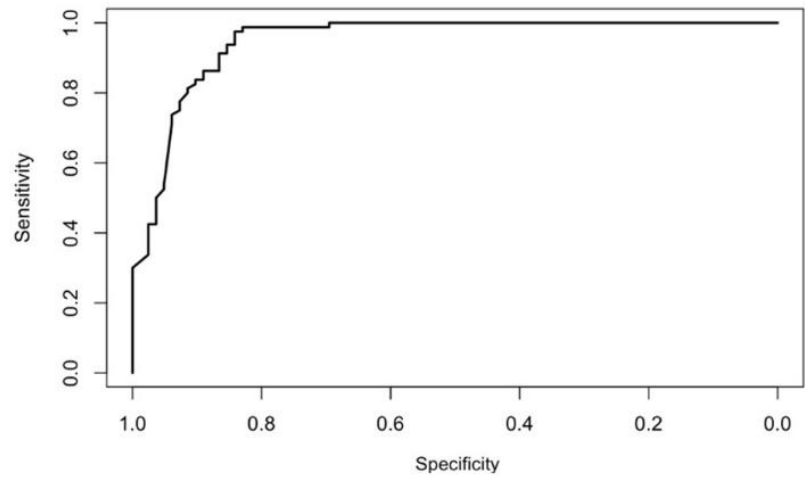

Figure 4. Random forest classification models describing the accuracy of statistical predictions given a discrimination threshold of A. 0.9 or B. 0.95 . 


\section{Decay modeling}

Independently, we developed a physical decay model to explain the shapes of the curves in Figure 2 and support our interpretation of the activity distribution on the radioTLC plate. An $\left[{ }^{225} \mathrm{Ac}\right] \mathrm{Ac}(\mathrm{OAc})_{3}$ control TLC plate allowed us to calibrate the model for non-heterogeneous migration of actinium-225, either as the triacetate salt or as the EDTA transchelation complex. This gave rise to four additional refinements of the basic model:

1. Based on our experiments, the amounts of $\left[{ }^{225} \mathrm{Ac}\right] \mathrm{Ac}-\mathrm{EDTA}$ and $\left[{ }^{213} \mathrm{Bi}\right] \mathrm{Bi}-\mathrm{EDTA}$ in the middle section sum to $41.3 \pm 14.4 \%$ of the total activity. For our model we will approximate the value to $40 \%$.

2. This implies that all labeled compounds, irrespective of structure, are located in grid section 1 (Figure $1 \mathrm{C}$ ) immediately after running the TLC, $40 \%$ of the metals not bound by the ligands (parent and daughter radionuclides that did not run homogeneously on the TLC plate) are in section 2, and the remaining $60 \%$ of these metals are in section 3 .

3. For ligands bearing macropa, $60 \%$ of the bismuth- 213 not bound by the ligands $(30 \%$ of the initial amount due to decay) is in section 3, and $40 \%$ (20\% following decay) is in section 2 immediately after running the TLC.

4. For ligands bearing DOTA or DOTA derivatives, the majority of bismuth-213 will be complexed by the ligands and retained in section 1. Small activities of daughter radionuclides will be observed in sections 2 and 3.

On this basis, activity in each TLC grid was expressed as a sum of the activities corresponding to actinium225 and bismuth-213. For the ligands RPS-072, EuK-107, and EuK-106, which contain DOTA or a DOTA derivative, the sums are described by the equations in Figure 5A. Activity in section 1 of the TLC is due to the contribution of the decay of bound actinium-225, the contribution of bismuth-213 generated from actinium-225 decay, and the contribution of bound bismuth-213 that was present as part of the initial equilibrium. The activity in section 2 is due to actinium-225 that was not bound by the ligands and did not run homogeneously on the TLC plate, and bismuth-213 generated by the decay of this actinium-225. Finally, the activity in section 3 is due to the bulk of the actinium-225 (which migrated with the solvent front) and the bismuth-213 produced by the decay of this actinium-225 (Figure 6).

\section{A}

$$
\begin{aligned}
& \text { Section 1: } A=\frac{3}{5.25} \cdot\left(A_{l} \cdot e^{-\lambda_{1} t_{1}}\right)+\frac{2.25}{5.25} A_{l} \frac{\lambda_{2}}{\lambda_{2}-\lambda_{1}}\left(e^{-\lambda_{1} t_{1}}-e^{-\lambda_{2} t_{2}}\right)+\frac{2.25}{5.25} A_{0} e^{-\lambda_{2} t_{2}} \\
& \text { Section 2: } A=\frac{3}{5.25} \cdot 0.4 \cdot\left(\left(100-A_{l}\right) \cdot e^{-\lambda_{1} t_{1}}\right)+\frac{2.25}{5.25} \cdot 0.4 \cdot\left(100-A_{l}\right) \frac{\lambda_{2}}{\lambda_{2}-\lambda_{1}}\left(e^{-\lambda_{1} t_{1}}-e^{-\lambda_{2} t_{2}}\right) \\
& \text { Section 3: } A=\frac{3}{5.25} \cdot 0.6 \cdot\left(\left(100-A_{l}\right) \cdot e^{-\lambda_{1} t_{1}}\right)+\frac{2.25}{5.25} \cdot 0.6 \cdot\left(100-A_{l}\right) \frac{\lambda_{2}}{\lambda_{2}-\lambda_{1}}\left(e^{-\lambda_{1} t_{1}}-e^{-\lambda_{2} t_{2}}\right) \\
& \text { B } \\
& \text { Section 1: } A=\frac{3}{5.25} \cdot\left(A_{l} \cdot e^{-\lambda_{1} t_{1}}\right)+\frac{2.25}{5.25} A_{l} \frac{\lambda_{2}}{\lambda_{2}-\lambda_{1}}\left(e^{-\lambda_{1} t_{1}}-e^{-\lambda_{2} t_{2}}\right) \\
& \text { Section 2: } A=\frac{3}{5.25} \cdot 0.4 \cdot\left(\left(100-A_{l}\right) \cdot e^{-\lambda_{1} t_{1}}\right)+\frac{2.25}{5.25} \cdot 0.4 \cdot\left(100-A_{l}\right) \frac{\lambda_{2}}{\lambda_{2}-\lambda_{1}}\left(e^{-\lambda_{1} t_{1}}-e^{-\lambda_{2} t_{2}}\right)+\frac{2.25}{5.25} \cdot 0.4 \cdot A_{0} e^{-\lambda_{2} t_{2}} \\
& \text { Section 3: } A=\frac{3}{5.25} \cdot 0.6 \cdot\left(\left(100-A_{l}\right) \cdot e^{-\lambda_{1} t_{1}}\right)+\frac{2.25}{5.25} \cdot 0.6 \cdot\left(100-A_{l}\right) \frac{\lambda_{2}}{\lambda_{2}-\lambda_{1}}\left(e^{-\lambda_{1} t_{1}}-e^{-\lambda_{2} t_{2}}\right)+\frac{2.25}{5.25} \cdot 0.6 \cdot A_{0} e^{-\lambda_{2} t_{2}}
\end{aligned}
$$

Figure 5. Equations describing the distribution of activity on the TLC plate. Sections are defined according to Figure 1C. Subscript 1 denotes actinium-225, subscript 2 denotes bismuth-213 and subscript 0 denotes 
initial conditions. A. Equations for RPS-072, EuK-106, and EuK-107. B. Equations for RPS-074, RPS-088, and RPS-092.

The equations describing macropa-containing ligands RPS-074, RPS-088, and RPS-092 are presented in Figure 5B. Activity in section 1 of the TLC is due to actinium-225 bound by the ligands and daughter radionuclides generated by decay of the parent actinium-225. The contributions to section 2 are actinium225 and bismuth-213 that were not bound by the ligands at the time of spotting and did not run homogeneously on the TLC plate, and the decay of the actinium-225 to its daughters. Activity in section 3 is due to the contribution of the bulk actinium-225, the bulk bismuth-213, and the daughter radionuclides generated by decay of actinium-225 (Figure 6).

\section{Macropa Chelator}

\section{DOTA Chelator}
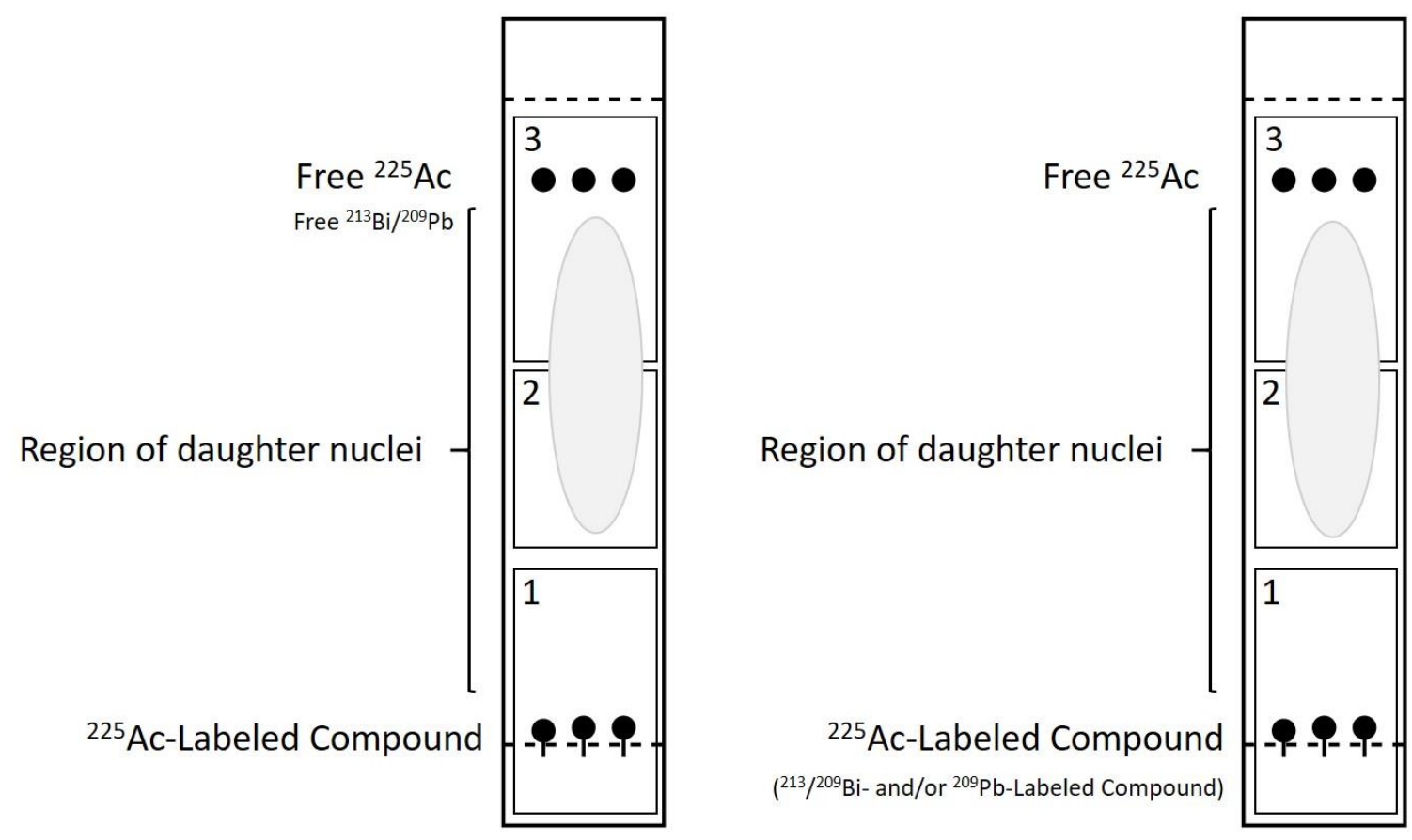

Figure 6. Schematic of the distribution of radiolabeled compounds, uncomplexed metal, and daughter nuclei ejected from the labeled compounds during radioactive decay.

The physical decay model supports our interpretation of the activity distribution on the TLC plate and rationalizes the shapes of the curves plotted in Figure 2. Early measurement of the DOTA-containing ligands overestimates the labeling yield when the true yield is $\leq 40 \%$ (Figure 7 ). When the yield is $\geq 80 \%$, however, early measurement underestimates the true value. This is largely due to discrepancies in TLC section 1 (Figure S6). Activity in TLC sections 2 and 3 is underestimated at early time points, but the difference between predicted yield and true yield is less than $3 \%$ over the $26 \mathrm{~h}$. These deviations from the true yield also arise in the modeled data, indicating that they are a consequence of the disruption of secular equilibrium rather than a systematic experimental error. 
By contrast, early measurement underestimates the labeling yield for macropa-containing ligands, particularly when true reaction yield is high ( $\geq 80 \%$ ) (Figure 7 ). In this case, the discrepancy is the result of overestimation of actinium-225 in sections 2 and 3 of the TLC grid (Figure S6). As secular equilibrium is approached, the differences between the experimentally-determined yield or the decay-modelled yield and the true yield reduce significantly, independent of the chelating moiety (Figure S7). The differences are statistically significant at the $30 \mathrm{~min}$ and $2 \mathrm{~h}$ analysis times $(\mathrm{p}<0.01)$, but not at later analyses $(p>$ 0.1) (Table S4).

A.
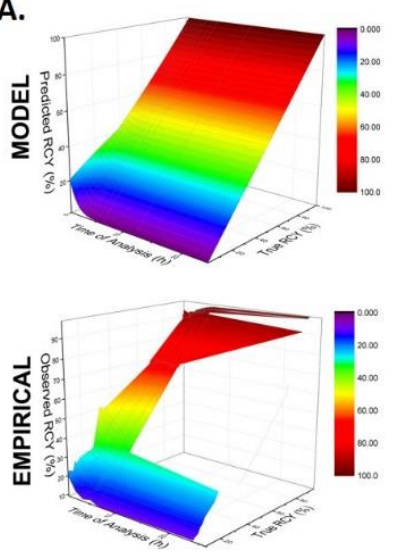
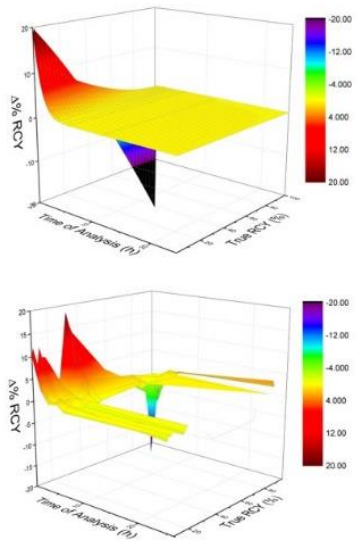

B.
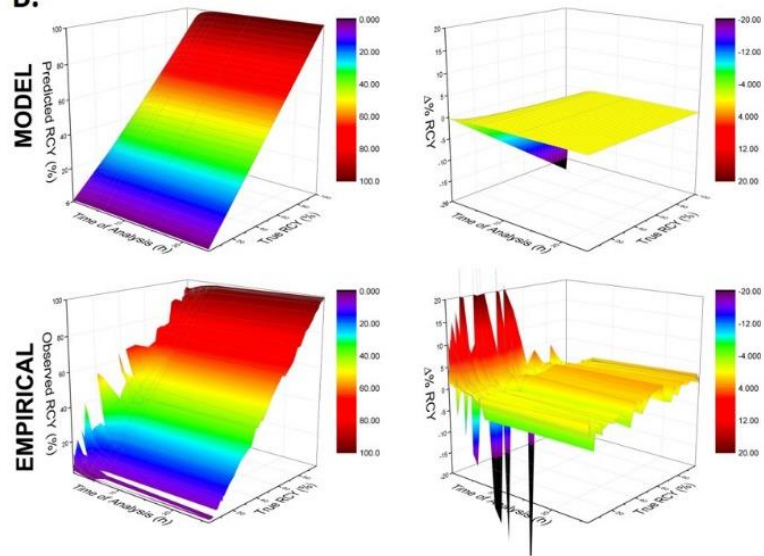

Figure 7. Comparison of the modeled (top) and empirical (bottom) yields against the true radiochemical yield for A. DOTA-containing compounds, and B. Macropa-containing compounds. In both panels, the left column compares the yield predicted by the decay model (Z-axis, top) or the empirically-measured yield ( $Z$-axis, bottom) against the true yield ( $Y$-axis) as a function of observation time (X-axis). The right column plots the difference between the modeled yield and true yield (Z-axis, top) or difference between the empirically-measured yield and true yield (Z-axis, bottom) as a function of true yield ( $\mathrm{Y}$-axis) and observation time (X-axis).

\section{Discussion}

Despite the long half-life of actinium-225, it is advantageous to be able to administer a ${ }^{225} \mathrm{Ac}$-labeled radiopharmaceutical as quickly as possible after radiosynthesis is completed. One advantage is the simplification of logistics: the radiopharmaceutical can be prepared and administered during normal business hours. Ideally, the radiolabeling is performed with high efficiency, allowing the reaction mixture to be buffered and directly administered to the patient without a purification step. It is therefore advantageous to determine as quickly as possible whether an additional purification step is required to remove uncomplexed actinium-225 prior to administration of the radiopharmaceutical. Finally, rapid administration better guarantees the purity of the radiopharmaceutical by virtue of minimizing the number of decays that occur while the compound is in its final formulated solution, thereby avoiding product decomposition through radiolysis. Each decay of actinium-225 results in the emission of one $\alpha$ particle, with simultaneous recoil of the francium-221 daughter nucleus. This recoil energy may be 
sufficient to liberate the daughter nucleus from the chelator into solution, potentially leading to the administration of non-targeted ionizing radiation to the patient (36). Furthermore, the $\alpha$-particle itself may induce radiolytic damage to the radiopharmaceutical. If this damage occurs to the targeting moiety of the ligand, it may reduce in vivo targeting and lead to further accumulation of radioactivity in nontarget tissue.

The desire to quickly release the production batch must not supersede the importance of accurately assessing the purity of the compound before administration. In the case of ${ }^{225} \mathrm{Ac}$-labeled radiopharmaceuticals, this can be accomplished by high-resolution $\gamma$-spectrometry $(11,23,37)$. As the $\alpha$ particle emissions cannot penetrate the detector, and actinium-225 does not itself decay by $\gamma$-emission, actinium-225 is indirectly detected and quantified via the $218 \mathrm{keV} \gamma$-emission of francium-221 $(11,23,37)$. Quantification is only possible after waiting at least $60 \mathrm{~min}$ for the equilibrium between actinium-225 and francium-221 to be reached (11).

An alternative strategy for quantification is to use a form of solid state detection, such as phosphorimaging. In this scenario, all particles are detected, and the sensitivity of the approach is enhanced. However, $\gamma$ particles are detected by the phosphor screen with a different efficiency than $\alpha$ and $\beta$ particles. In addition, the disruption of the secular equilibrium further complicates the analysis of radiochemical purity. To further complicate the analysis, an initial activity of $37 \mathrm{MBq}$ actinium-225 reaches secular equilibrium with francium-221 after $55 \mathrm{~min}$ and with bismuth-213 after approximately $6.5 \mathrm{~h}$ (38). On this basis, we analyzed the TLC plates $6.5 \mathrm{~h}$ and $26 \mathrm{~h}$ after they were run, a time point at which actinium-225 is in secular equilibrium with all of its daughter radionuclides. We selected four earlier time points, separated by 90 min intervals in order to investigate meaningfully different QC scenarios - waiting $30 \mathrm{~min}$ or $60 \mathrm{~min}$ after running the TLC may not have a major impact on release testing, but waiting $2 \mathrm{~h}$ rather than 30 min may be more significant. These intervals also accommodated the logistical constraints imposed by the experiments. In total, we made 585 observations spanning labeling yields from $1.8 \%$ to $99.5 \%$. The measurements were more heavily distributed toward the extremes, with $42 \%$ of observations lying in the range $90-100 \%$, and $21 \%$ lying in the range $0-20 \%$ (Figure S7). It is likely that the unequal distribution of measurements influenced our models. However, with protocols for radiolabeling DOTA and macropa with actinium-225 now well established, a dataset weighed heavily by yields $>90 \%$ is consistent with the typical radiopharmacy experience.

Our decay model broadly rationalizes the distribution of activities that we observed on the TLC plates. Deviations between both modeled yields and empirically-measured yields and the true yield were substantial during the first $2 \mathrm{~h}$ of analysis before the values converged as secular equilibrium was approached (Figure 7). In general, the modeled values and measured values closely agree: the values differ by $<3 \%$ after $2 \mathrm{~h}$. These differences are likely the result of a combination of the underlying simplifications of the model. In particular, the model does not consider the contribution of daughter nuclei emitted during decays of actinium-225 (or bismuth-213) that occur while the TLC plate is running in the mobile phase. These daughter nuclei will migrate up the TLC plate but will not reach the solvent front because they were generated later than the uncomplexed metal (Figure 6). The model therefore overestimates the rate of decay of radioactivity from the TLC grid sections. Although experimental measurements were used to calibrate the decay model, ultimately the model only requires adequate 
separation of radiolabeled compounds from uncomplexed metals. This suggests that our results are generalizable to any suitable radioTLC method.

Our initial iteration of the statistical modeling assigned equal weights to each measurement. The fit is generally good (Figure S4). As the aim of these studies is to arrive at a model that can be used primarily to assess the radiochemical purity of mixtures in the $90-100 \%$ range, we explored a model in which measurements in this region were given extra weight. This is because reactions with true purity $<90 \%$ will likely require purification prior to administration, meaning that highly accurate quantification of the yield is only necessary for purities $>90 \%$. We therefore assigned a weight of 10 times more to each observation $>90 \%$. The result is a model that fit slightly better in upper extreme, and less well in other regions. The weighted model predicts slightly higher true yields for measurements $>90 \%$ (Table S2), but both models demonstrate similar MAE (Tables S3). As these predictions may prevent unnecessary purification procedures to be performed and reduce analysis time, we validated our weighted model against an independent data set. Predictive accuracy was extremely high ( $\pm 1 \%$ ) (Figure S8). By contrast, accuracy of the non-weighted model was slightly lower $( \pm 3 \%)$. This suggests that our choice of predictive statistical model is justified.

The mean average error of our model decreases substantially from $30 \mathrm{~min}$ to $2 \mathrm{~h}$. This implies that radiochemical purity can be more accurately predicted by analyzing the TLC plate at least $2 \mathrm{~h}$ after it is removed from the mobile phase. Quantification of yield at earlier time points may over- or underestimate the true yield depending on the chelating moiety. This is because of the different affinities that the chelators have for the daughter radionuclides of actinium-225. For reactions performed at $95{ }^{\circ} \mathrm{C}$ using DOTA, bismuth-213 present in the reaction mixture will be complexed and contribute to the counts detected in grid section 1 of the TLC plate. At early analysis time points, therefore, the activity of the ${ }^{225} A c-$ labeled compound may be overestimated. It is likely that chelation of bismuth-213 may increase relative to actinium-225 at lower temperatures due to more rapid labeling kinetics (39), increasing the degree to which early measurements may be misleading. By contrast, bismuth-213 present in the reaction mixture will be incompletely complexed by macropa at any reaction temperature. Consequently it will migrate along with any actinium-225 that is not bound to the ligands, leading to an underestimation of radiochemical yield.

\section{Conclusions}

As TAT with actinium-225 continues to demonstrate promise under clinical evaluation, there is a growing need to standardize QC procedures for ${ }^{225} \mathrm{Ac}$-labeled radiopharmaceuticals. A major challenge is the ability to accurately quantify radiochemical purity given the time required for actinium-225 to reach secular equilibrium. We compiled a large dataset of empirically-measured radiolabeling yields using multiple radioligands conjugated to DOTA, derivatives of DOTA, or macropa by collecting measurements at various times after running a TLC. Our generalized model confirms that predictive accuracy improves after $30 \mathrm{~min}$, and is comparable at all time points from $2 \mathrm{~h}$ to $6 \mathrm{~h}$. On this basis we argue that the $2 \mathrm{~h}$ analysis time point best balances the need to accurately assess the purity of the radiopharmaceutical with the need to release it for administration as quickly as possible. 


\section{List of Abbreviations}

3D: Three dimensional

3p-C-DEPA: 2-[(Carboxymethyl)][5-(4-nitrophenyl-1-[4,7,10-tris(carboxymethyl)-1,4,7,10-

tetraazacyclododecan-1-yl]pentan-2-yl)amino]acetic acid

AUC: Area under the curve

$\mathrm{Cl}$ : Confidence interval

DMSO: Dimethylsulfoxide

DOTA: 1,4,7,10-Tetraazacyclododecane-1,4,7,10-tetracetic acid

EDTA: Ethylenediaminetetraacetic acid

Macropa: N,N'-bis[(6-carboxy-2-pyridyl)methyl]-4,13-diaza-18-crown-6

MAE: Mean average error

$\mathrm{MeOH}:$ Methanol

OAc: Acetate

PSMA: Prostate-specific membrane antigen

QC: Quality control

RCP: Radiochemical purity

$\mathrm{RCY}$ : Radiochemical yield

$\mathrm{R}_{\mathrm{f}}$ : Retention factor

ROC: Receiver operating characteristics

TAT: Targeted alpha-particle therapy

TLC: Thin layer chromatography

\section{Declarations}

Ethics Approval and Consent to Participate:

Not applicable.

Consent for Publication: 
Not applicable.

Availability of Data and Material:

All data generated during the experiments is reported in the manuscript. Raw data is available from the authors upon request.

Competing Interests:

JMK, AA, and JWB hold intellectual property rights related to compounds described in this manuscript. Additionally, JMK, AA, and JWB hold equity in Noria Therapeutics, Inc, which has licensed technology related to these compounds.

Funding:

Not applicable.

\section{Authors' Contributions:}

JMK designed the study, performed the radiolabeling experiments, and wrote the manuscript. AA performed the decay modeling, provided figures for the manuscript, and contributed to the manuscript revisions. ES performed the statistical modeling, provided figures for the manuscript, and contributed to the manuscript revisions. JWB contributed to the study design and to the manuscript revisions. All authors read and approved the final manuscript.

\section{Acknowledgments:}

The authors wish to acknowledge Dr. Jay Tinklepaugh for assistance with the acquisition of radioTLC images and for supplying the independent data set with which the statistical model was validated.

\section{References}

1. Nilsson S, Larsen RH, Fosså SD, et al. First Clinical Experience with $\alpha$-Emitting Radium-223 in the Treatment of Skeletal Metastases. Clin Cancer Res. 2005;11:4451-4459.

2. Nilsson S, Strang P, Aksnes AK, et al. A randomized, dose-response, multicenter phase II study of radium-223 chloride for the palliation of painful bone metastases in patients with castration-resistant prostate cancer. Eur J Cancer. 2012;48:678-686.

3. Nilsson S, Cislo P, Sartor O, et al. Patient-reported quality-of-life analysis of radium-223 dichloride from the phase III ALSYMPCA study. Ann Oncol. 2016;27:868-874.

4. Zalutsky MR, Reardon DA, Akabani G, et al. Clinical Experience with alpha-Particle Emitting ${ }^{211} \mathrm{At}$ : Treatment of Recurrent Brain Tumor Patients with ${ }^{211}$ At-Labeled Chimeric Antitenascin Monoclonal Antibody 81C6. J Nucl Med. 2008;49:30-38. 
5. Hallqvist A, Bergmark K, Bäck T, et al. Intraperitoneal $\alpha$-Emitting Radioimmunotherapy with ${ }^{211}$ At in Relapsed Ovarian Cancer: Long-Term Follow-up with Individual Absorbed Dose Estimations. J Nucl Med. 2019;60:1073-1079.

6. Meredith RF, Torgue JJ, Rozgaja TA, et al. Safety and Outcome Measures of First-in-Human Intraperitoneal $\alpha$ Radioimmunotherapy with ${ }^{212} \mathrm{~Pb}-\mathrm{TCMC}-$ Trastuzumab. Am J Clin Oncol. 2018;41:716-721.

7. Rosenblat TL, McDevitt MR, Mulford DA, et al. Sequential Cytarabine and $\alpha$-Particle Immunotherapy with Bismuth-213-Lintuzumab (HuM195) for Acute Myeloid Leukemia. Clin Cancer Res. 2010;16:5303-5311.

8. Cordier D, Forrer F, Bruchertseifer F, et al. Targeted alpha-radionuclide therapy of functionally critically located gliomas with ${ }^{213} \mathrm{Bi}-\mathrm{DOTA}-\left[\mathrm{Thi}^{8}, \mathrm{Met}\left(\mathrm{O}_{2}\right)^{11}\right]$-substance $\mathrm{P}$ : a pilot trial. Eur J Nucl Med Mol Imaging. 2010;37:1335-1344.

9. Kratochwil C, Giesel FL, Bruchertseifer F, et al. ${ }^{213}$ Bi-DOTATOC receptor-targeted alpharadionuclide therapy induces remission in neuroendocrine tumours refractory to beta radiation: a first-inhuman experience. Eur J Nucl Med Mol Imaging. 2014;41:2106-2119.

10. Juric JG, Levy MY, Park JH, et al. Phase I Trial of $\alpha$-Particle Therapy with Actinium-225 $\left({ }^{225} \mathrm{Ac}\right)$ Lintuzumab (anti-CD33) and Low-Dose Cytarabine (LDAC) in Older Patients with Untreated Acute Myeloid Leukemia (AML.). Blood. 2016;128:4050.

11. Kratochwil C, Bruchertseifer F, Giesel FL, et al. ${ }^{225}$ Ac-PSMA-617 for PSMA-Targeted $\alpha$-Radiation Therapy of Metastatic Castration-Resistant Prostate Cancer. J Nucl Med. 2016;57:1941-1944.

12. Scheinberg DA, McDevit MR. Actinium-225 in targeted alpha-particle therapeutic applications. Curr Radiopharm. 2011;4:306-320.

13. Morgenstern A, Apostolidis C, Kratochwil C, Sathekge M, Krolicki L, Bruchertseifer F. An Overview of Targeted Alpha Therapy with ${ }^{225}$ Actinium and ${ }^{213}$ Bismuth. Curr Radiopharm. 2018;11:200-208.

14. Robertson AKH, Ramogida CF, Schaffer P, Radchenko V. Development of ${ }^{225} \mathrm{Ac}$ Radiopharmaceuticals: TRIUMF Perspectives and Experiences. Curr Radiopharm. 2018;11:156-172.

15. Kratochwil C, Bruchertseifer F, Giesel F, Apostolidis C, Haberkorn U, Morgenstern A. Ac-225DOTATOC - an empiric dose finding for alpha emitter based radionuclide therapy of neuroendocrine tumors. J Nucl Med. 2015;56:1232. 
16. Kratochwil $\mathrm{C}$, Bruchertseifer $\mathrm{F}$, Rathke $\mathrm{H}$, et al. Targeted $\alpha$-Therapy of Metastatic CastrationResistant Prostate Cancer with ${ }^{225}$ Ac-PSMA-617: Swimmer-Plot Analysis Suggests Efficacy Regarding Duration of Tumor Control. J Nucl Med. 2018;59:795-802.

17. Sathekge M, Bruchertseifer F, Knoesen O, et al. ${ }^{225}$ Ac-PSMA-617 in chemotherapy-naive patients with advanced prostate cancer: a pilot study. Eur J Nucl Med Mol Imaging. 2019;46:129-138.

18. Khreish F, Ebert N, Ries M, et al. ${ }^{225}$ Ac-PSMA-617/ $/{ }^{177}$ Lu-PSMA-617 tandem therapy of metastatic castration-resistant prostate cancer: pilot experience. Eur J Nucl Med Mol Imaging. 2020;47:721-728.

19. Krolicki L, Bruchertseifer F, Morgenstern A, et al. Safety and Therapeutic Efficacy of ${ }^{225}$ Ac-DOTASubstance P for Therapy of Brain Tumors. J Med Imaging Radiat Sci. 2019;50:S22.

20. Tagawa ST, Vallabhajosula S, Jhanwar Y, et al. Phase I dose-escalation study of ${ }^{225} \mathrm{Ac}-J 591$ for progressive metastatic castration resistant prostate cancer (mCRPC). J Clin Oncol. 2018;36:TPS399.

21. McDevitt MR, Ma D, Simon J, Frank RK, Scheinberg DA. Design and synthesis of ${ }^{225} A c$ radioimmunopharmaceuticals. Appl Radiat Isot. 2002;57:841-847.

22. Thiele $\mathrm{NH}$, Brown V, Kelly JM, et al. An Eighteen-Membered Macrocyclic Ligand for Actinium-225 Targeted Alpha Therapy. Angew Chem Int Ed. 2017;56:14712-14717.

23. Ramogida CF, Robertson AKH, Jermilova U, et al. Evaluation of polydentate picolinic acid chelating ligands and an $\alpha$-melanocyte-stimulating hormone derivative for targeted alpha therapy using ISOLproduced ${ }^{225}$ Ac. EJNMMI Radiopharm Chem. 2019;4:21.

24. Poty S, Membreno R, Glaser JM, et al. The inverse electron-demand Diels-Alder reaction as a new methodology for the synthesis of ${ }^{225} \mathrm{Ac}$-labelled radioimmunoconjugates. Chem Commun (Camb). 2018;54:2599-2602.

25. Kruijff RMd, Raavé R, Kip A, et al. The in vivo fate of ${ }^{225} \mathrm{Ac}$ daughter nuclides using polymersomes as a model carrier. Sci Rep. 2019;9:11671.

26. Shukurov R, Veliyev M, Dadashov Z, Isayev J, Novruzov F. Labeling process and quality control results of ${ }^{225}$ Ac-PSMA-617 for targeted alpha particle therapy for metastatic prostate cancer. J Nucl Med. 2019;60:1611.

27. Deal KA, Davis IA, Mirzandeh S, Kennel SJ, Brechbiel MW. Improved In Vivo Stability of Actinium225 Macrocyclic Complexes. J Med Chem. 1999;42:2988-2992. 
28. Kelly JM, Amor-Coarasa A, Ponnala S, et al. Albumin-Binding PSMA Ligands: Implications for Expanding the Therapeutic Window. J Nucl Med. 2019;60:656-663.

29. Kelly JM, Amor-Coarasa A, Ponnala S, et al. A Single Dose of ${ }^{225}$ Ac-RPS-074 Induces a Complete Tumor Response in an LNCaP Xenograft Model. J Nucl Med. 2019;60.

30. Kelly JM, Amor-Coarasa A, Nikolopoulou A, et al. Assessment of PSMA targeting ligands bearing novel chelates with application to theranostics: Stability and complexation kinetics of ${ }^{68} \mathrm{Ga}^{3+},{ }^{111} \mathrm{In}^{3+},{ }^{177} \mathrm{Lu}^{3+}$ and ${ }^{225} \mathrm{Ac}^{3+}$. Nucl Med Biol. 2017;55:38-46.

31. Ho TK. Random decision forests. Paper presented at: 3rd International Conference on Document Analysis and Recognition; 14-16 Aug, 1995; Montreal, CA.

32. Liaw A, Wiener M. Classification and regression by randomForest. $R$ news. 2002;2:18-22.

33. Baranyai Z, Tircsó G, Rösch F. The Use of the Macrocyclic Chelator DOTA in Radiochemical Separations. Eur J Inorg Chem. 2019:36-56.

34. Khabibullin AR, Karolak A, Budzevich MM, McLaughlin ML, Morse DL, Woods LM. Structure and properties of DOTA-chelated radiopharmaceuticals within the ${ }^{225}$ Ac decay pathway. Med Chem Commun. 2018;9:1155-1163.

35. Thiele NA, Wilson JJ. Implementing f-Block Metal lons in Medicine: Tuning the Size Selectivity of Expanded Macrocycles. Inorg Chem. 2019;58:10483-10500.

36. Kruijff RMd, Wolterbeek HT, Denkova AG. A Critical Reviw of Alpha Radionuclide Therapy - How to Deal with Recoiling Daughters? Pharmaceuticals. 2015;8:321-336.

37. Robertson AKH, Ramogida CF, Rodríguez-Rodríguez $C$, et al. Multi-isotope SPECT imaging of the 225Ac decay chain: feasibility studies. Phys Med Biol. 2017;62:4406-4420.

38. Tichacek CJ, Budzevich MM, Wadas TJ, Morse DL, Moros EG. A Monte Carlo Method for Determining the Reesponse Relationship between Two Commonly Used Detectors to Indirectly Measure Alpha Particle Radiation Activity. Molecules. 2019;24:3397.

39. Song HA, Kang CS, Baidoo KE, et al. An Efficient Bifunctional Decadentate Ligand 3p-C-DEPA for Targeted Alpha Radioimmunotherapy Applications. Bioconjug Chem. 2011;22:1128-1135. 


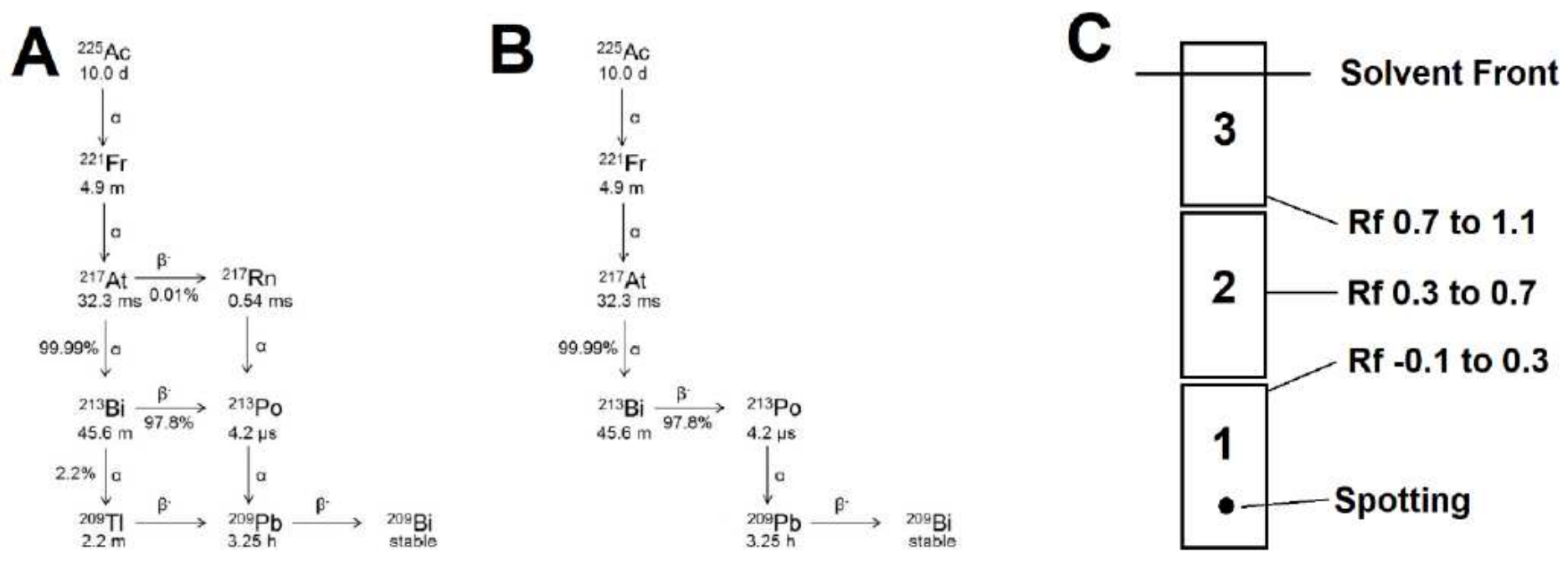

\section{Figure 1}

Approximations used for the mathematical model. A. Actinium-225 decay scheme. B. The actinium-225 decay scheme used for the purpose of the model. C. General representation of the box grid used to quantify radiolabeling by radioTLC

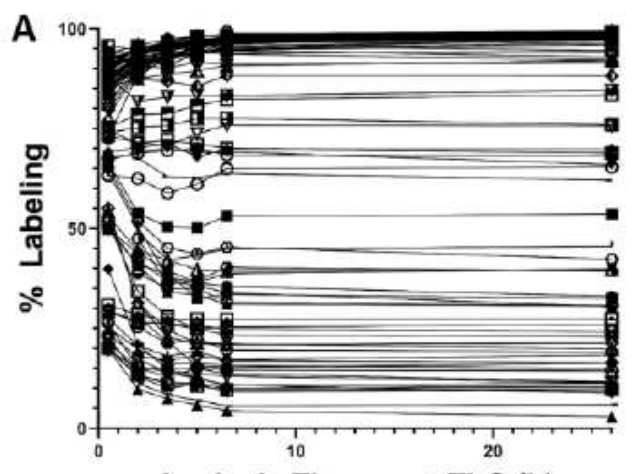

Analysis Time post-TLC (h)

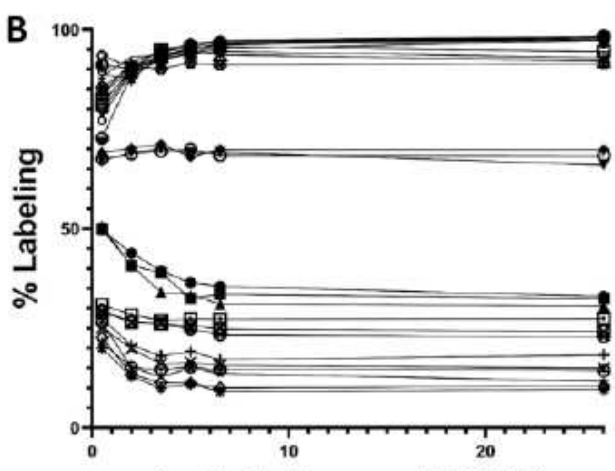

Analysis Time post-TLC (h)

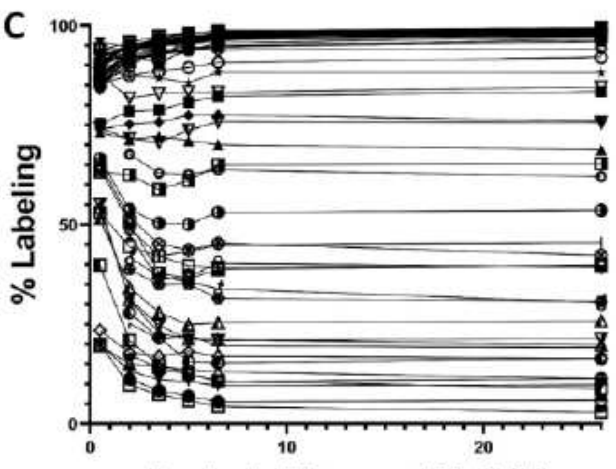

Analysis Time post-TLC (h)

Figure 2

Figure 2. Plot of experimentally-derived radiolabeling versus analysis time after TLC for multiple reaction conditions. Radiolabeling was performed using RPS-072, RPS-074, RPS-088, RPS-092, EuK-106, or EuK107 and [225Ac] AcCl3 in $1 \mathrm{M} \mathrm{NH4OAc}$. Conditions are described in Table 1. Activity distribution on the TLC plates was visualized at the specified times using a Cyclone Plus Storage Phosphor System. Quantification of labeling was performed using OptiQuant ${ }^{\text {TM }}$ software. A. All Compounds. B. DOTAcontaining ligands (RPS-072, EuK-106, EuK-107). C. Macropa-containing ligands (RPS-074, RPS-088, RPS092). 

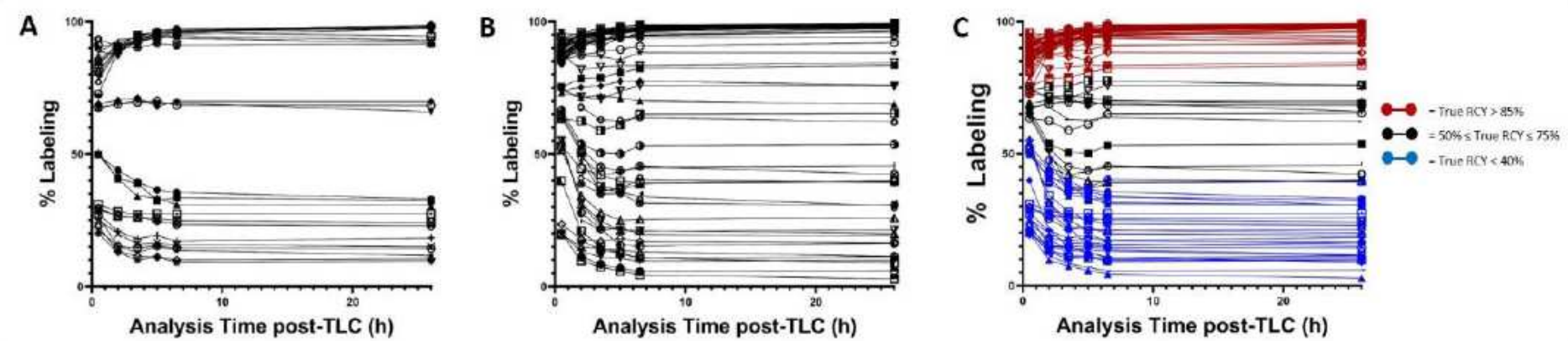

[AA1]

\section{Figure 3}

Figure 2. Plot of experimentally-derived radiolabeling versus analysis time after TLC for multiple reaction conditions. Radiolabeling was performed using RPS-072, RPS-074, RPS-088, RPS-092, EuK-106, or EuK107 and [225Ac] AcCl3 in $1 \mathrm{M} \mathrm{NH} 40 \mathrm{Ac}$. Conditions are described in Table 1. Activity distribution on the TLC plates was visualized at the specified times using a Cyclone Plus Storage Phosphor System.

Quantification of labeling was performed using OptiQuant ${ }^{\text {TM }}$ software. A. DOTA-containing ligands (RPS072, EuK-106, EuK-107). B. Macropa-containing ligands (RPS-074, RPS-088, RPS-092). C. All compounds.

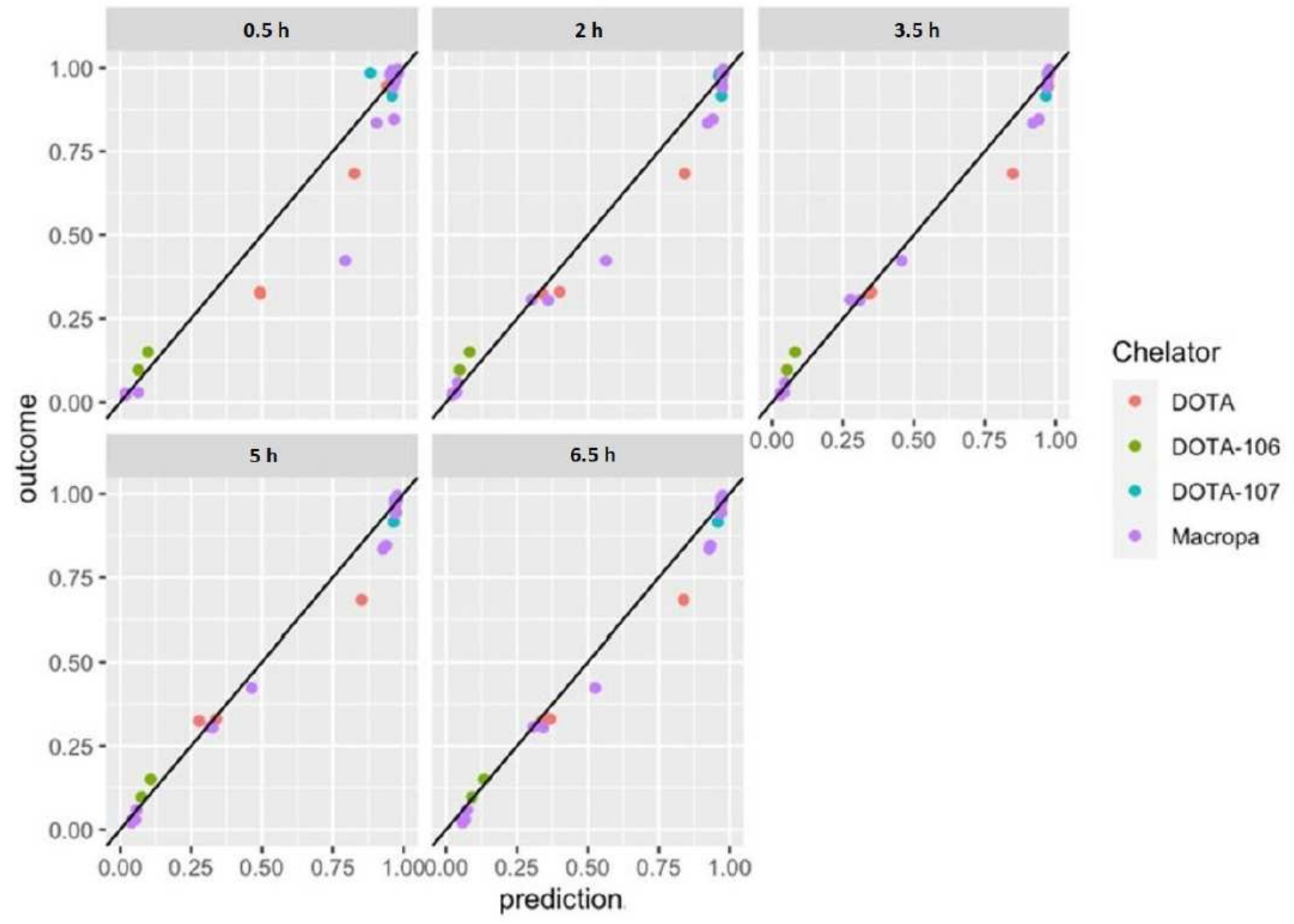




\section{Figure 4}

Figure 3. Predictive accuracy of QC measurements taken at $0.5 \mathrm{~h}, 2 \mathrm{~h}, 3.5 \mathrm{~h}, 5 \mathrm{~h}$, or $6.5 \mathrm{~h}$ after running the radioTLC plate. X-axis: Predicted radiochemical yield. Y-axis: True radiochemical yield, defined as the radiochemical yield when measured at $26 \mathrm{~h}$ after running the TLC.

A.

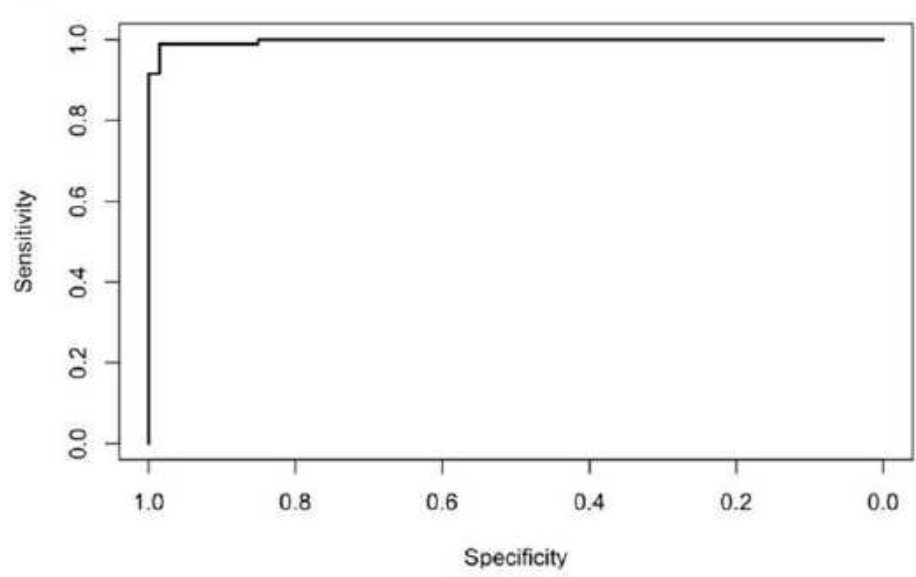

B.

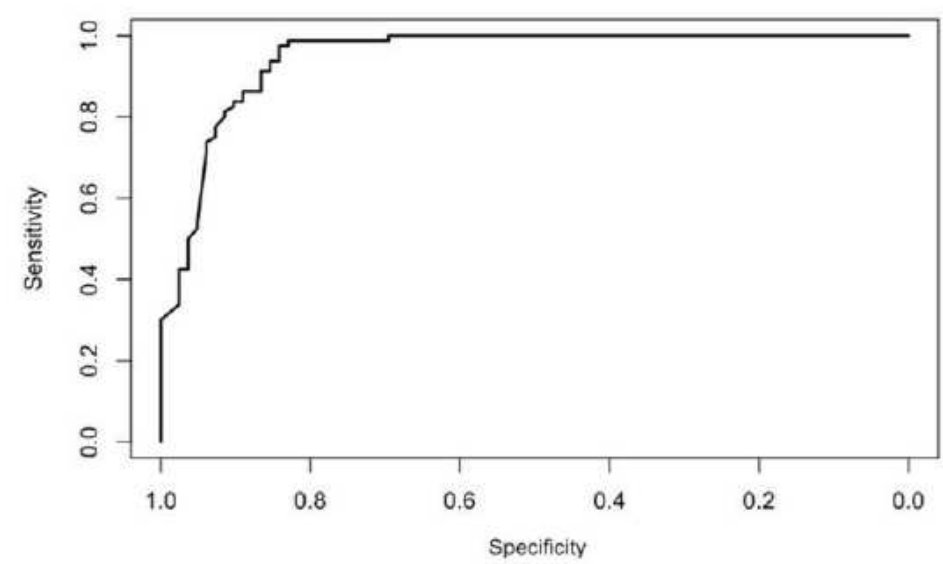

Figure 5

Figure 4. Random forest classification models describing the accuracy of statistical predictions given a discrimination threshold of A. 0.9 or B. 0.95 .

\section{A}

Section 1: $A=\frac{3}{5.25} \cdot\left(A_{l} \cdot e^{-\lambda_{1} t_{1}}\right)+\frac{2.25}{5.25} A_{l} \frac{\lambda_{2}}{\lambda_{2}-\lambda_{1}}\left(e^{-\lambda_{1} t_{1}}-e^{-\lambda_{2} t_{2}}\right)+\frac{2.25}{5.25} A_{0} e^{-\lambda_{2} t_{2}}$

Section 2: $A=\frac{3}{5.25} \cdot 0.4 \cdot\left(\left(100-A_{l}\right) \cdot e^{-\lambda_{1} t_{1}}\right)+\frac{2.25}{5.25} \cdot 0.4 \cdot\left(100-A_{l}\right) \frac{\lambda_{2}}{\lambda_{2}-\lambda_{1}}\left(e^{-\lambda_{1} t_{1}}-e^{-\lambda_{2} t_{2}}\right)$

Section 3: $A=\frac{3}{5.25} \cdot 0.6 \cdot\left(\left(100-A_{l}\right) \cdot e^{-\lambda_{1} t_{1}}\right)+\frac{2.25}{5.25} \cdot 0.6 \cdot\left(100-A_{l}\right) \frac{\lambda_{2}}{\lambda_{2}-\lambda_{1}}\left(e^{-\lambda_{1} t_{1}}-e^{-\lambda_{2} t_{2}}\right)$

B

Section 1: $A=\frac{3}{5.25} \cdot\left(A_{l} \cdot e^{-\lambda_{1} t_{1}}\right)+\frac{2.25}{5.25} A_{l} \frac{\lambda_{2}}{\lambda_{2}-\lambda_{1}}\left(e^{-\lambda_{1} t_{1}}-e^{-\lambda_{2} t_{2}}\right)$

Section 2: $A=\frac{3}{5.25} \cdot 0.4 \cdot\left(\left(100-A_{l}\right) \cdot e^{-\lambda_{1} t_{1}}\right)+\frac{2.25}{5.25} \cdot 0.4 \cdot\left(100-A_{l}\right) \frac{\lambda_{2}}{\lambda_{2}-\lambda_{1}}\left(e^{-\lambda_{1} t_{1}}-e^{-\lambda_{2} t_{2}}\right)+\frac{2.25}{5.25} \cdot 0.4 \cdot A_{0} e^{-\lambda_{2} t_{2}}$

Section 3: $A=\frac{3}{5.25} \cdot 0.6 \cdot\left(\left(100-A_{l}\right) \cdot e^{-\lambda_{1} t_{1}}\right)+\frac{2.25}{5.25} \cdot 0.6 \cdot\left(100-A_{l}\right) \frac{\lambda_{2}}{\lambda_{2}-\lambda_{1}}\left(e^{-\lambda_{1} t_{1}}-e^{-\lambda_{2} t_{2}}\right)+\frac{2.25}{5.25} \cdot 0.6 \cdot A_{0} e^{-\lambda_{2} t_{2}}$

\section{Figure 6}

Figure 5. Equations describing the distribution of activity on the TLC plate. Sections are defined according to Figure 1C. Subscript 1 denotes actinium-225, subscript 2 denotes bismuth-213 and subscript 0 denotes initial conditions. A. Equations for RPS-072, EuK-106, and EuK-107. B. Equations for RPS-074, RPS-088, and RPS-092. 

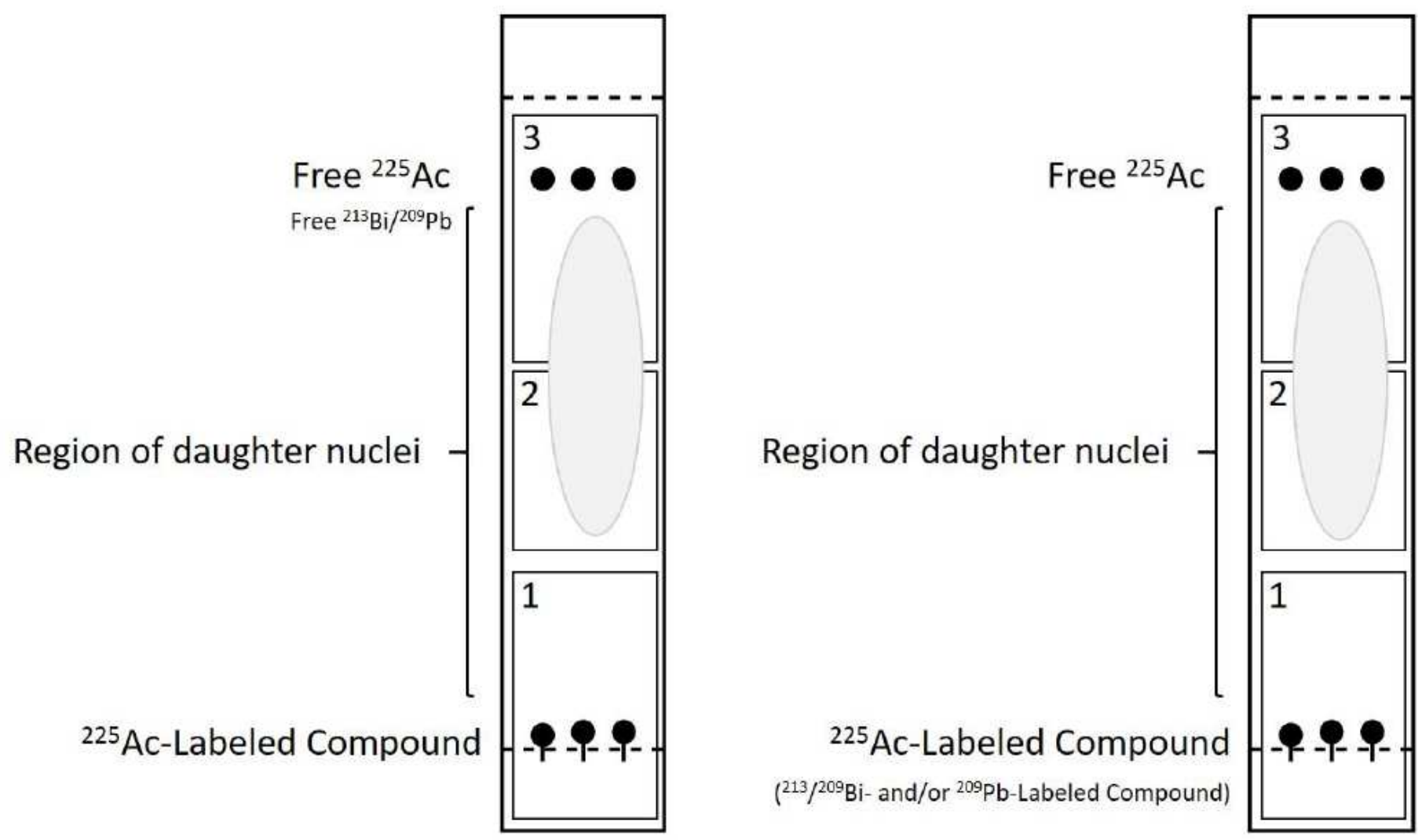

Figure 7

Figure 6. Schematic of the distribution of radiolabeled compounds, uncomplexed metal, and daughter nuclei ejected from the labeled compounds during radioactive decay.

A.
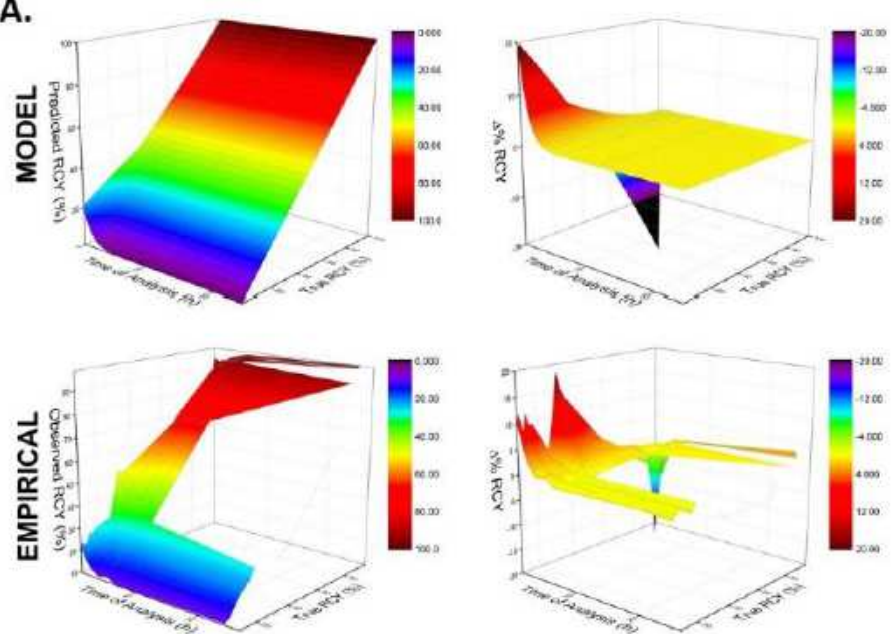

B.
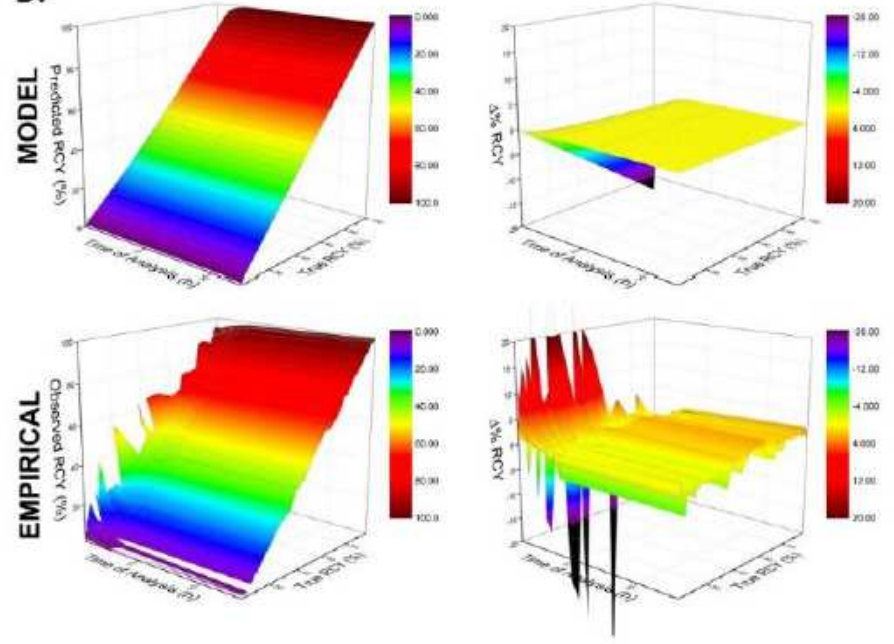

Figure 8 
Figure 7. Comparison of the modeled (top) and empirical (bottom) yields against the true radiochemical yield for A. DOTA-containing compounds, and B. Macropa-containing compounds. In both panels, the left column compares the yield predicted by the decay model (Z-axis, top) or the empirically-measured yield (Z-axis, bottom) against the true yield ( $Y$-axis) as a function of observation time (X-axis). The right column plots the difference between the modeled yield and true yield (Z-axis, top) or difference between the empirically-measured yield and true yield ( $Z$-axis, bottom) as a function of true yield ( $Y$-axis) and observation time (X-axis).

\section{Supplementary Files}

This is a list of supplementary files associated with this preprint. Click to download.

- Supplementallnformation.pdf 